\title{
Differentielle Beziehungsgestaltung in der Verhaltenstherapie: Auf dem Weg zu einer evidenzbasierten individualisierten Beziehungsgestaltung
}

\author{
Simon Bollmann ${ }^{a}$ Stephan Köhler ${ }^{b} \quad$ Anne Guhn ${ }^{b}$ Isabel Schamong ${ }^{a}$ \\ Philipp Sterzer $^{\mathrm{b}} \quad$ Eva-Lotta Brakemeier ${ }^{\mathrm{a}, \mathrm{c}}$

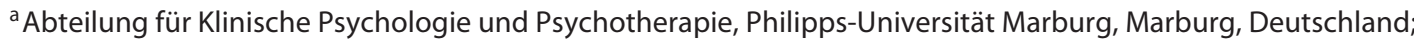 \\ ${ }^{b}$ Klinik für Psychiatrie und Psychotherapie, Campus Charité Mitte, Charité - Universitätsmedizin Berlin, Berlin, \\ Deutschland; ' Abteilung für Klinische Psychologie und Psychotherapie, Universität Greifswald, Greifswald, \\ Deutschland
}

\author{
Schlüsselwörter \\ Therapeutische Beziehungsgestaltung • \\ Therapeutische Allianz · Therapiephasen - Bedürfnisse · \\ Kindesmisshandlung
}

\section{Zusammenfassung}

Im Zuge der Anfänge der Verhaltenstherapie (VT) und der "kognitiven Wende" wurde der aktiven Beziehungsgestaltung und damit verbundenen Faktoren zunächst nur zweitrangig Beachtung geschenkt, obwohl die Bedeutung der Beziehung zwischen Therapeut und Patient auch in der VT bereits relativ früh erkannt wurde. Seitdem hat sich die VT substanziell weiterentwickelt. Insbesondere die aktive Gestaltung der therapeutischen Beziehung in Abhängigkeit verschiedener Variablen ist mittlerweile fester Bestandteil einer jeden VT. In dieser Übersichtsarbeit werden Konzepte der Beziehungsgestaltung dargestellt, welche der VT zugerechnet und im Rahmen der Ausbildung im deutschsprachigen Raum gelehrt werden. Fokussiert wird auf folgende drei Ansatzpunkte: Die Ausrichtung der Beziehung an (1) Therapiephasen, (2) Bedürfnissen, Motiven und Erwartungen sowie (3) frühen traumatisierenden Beziehungserfahrungen. Die aktuelle Studienlage legt nahe, dass pauschalisierte Handlungsempfehlungen zur aktiven Gestaltung der therapeutischen Beziehung zu kurz greifen. Ausgehend von der individuellen Problematik des einzelnen
Patienten wird die Realisierung der therapeutischen Beziehung im Sinne einer differentiellen Beziehungsgestaltung beschrieben und diskutiert.

(C) 2021 The Author(s) Published by S. Karger AG, Basel

\section{Keywords}

Therapeutic relationship formation - Therapeutic alliance . Phases of therapy · Needs · Childhood maltreatment

\begin{abstract}
Initially, the role of therapeutic relationship and associated factors was almost completely ignored in the early days of behavioral therapy (BT) and the "cognitive revolution". However, the importance of the relationship between therapist and patient - especially in clinical daily routine - was also recognized relatively early in BT. Since then, BT has developed substantially. In particular, the active shaping of the therapeutic relationship as a function of various variables has become an integral part of every $\mathrm{BT}$. In this review, concepts of relationship formation are
\end{abstract} mercial purposes requires written permission. 
presented that are attributed to cognitive-behavioral therapy and are taught as part of the psychotherapy training in German-speaking countries, focusing on the following three aspects: the orientation of the relationship towards (1) therapy phases, (2) needs, motives and expectations, and (3) childhood maltreatment. The current state of research suggests that generalized recommendations for action do not go far enough to actively shape the therapeutic relationship. Based on the individual problems of each patient, the realization of the therapeutic relationship in the sense of a differential relationship is described and discussed.

(c) 2021 The Author(s)

Published by S. Karger AG, Basel

\section{Hintergrund}

Die Frage nach der Bedeutung der therapeutischen Beziehung und ihrer Gestaltung für den psychotherapeutischen Behandlungsprozess ist bereits seit den Anfängen der Psychotherapie Gegenstand der Diskussion in Theorie und Praxis. Aus historischer Perspektive sind in diesem Kontext die Verdienste von psychoanalytisch-psychodynamischen sowie humanistisch orientierten Vertretern zur integrativen Konzeptualisierung und Evidenz der therapeutischen Beziehung besonders hervorzuheben. Die dazugehörige Debatte über die Bedeutung der therapeutischen Beziehung wird jedoch bis heute teilweise sehr kontrovers geführt. Deutlich wird dies besonders bei der Betrachtung der stellenweise sehr unterschiedlich definierten Konstrukte, welche die Komplexität der therapeutischen Beziehung entweder als Ganzes oder als Teilaspekt abzubilden versuchen. Schulenübergreifende Modelle zur Psychotherapie und ihrer Wirkungsweise betonen den hohen Stellenwert der therapeutischen Beziehung für eine erfolgreiche Psychotherapie [Frank und Frank, 1993; Orlinsky und Howard, 1987; Grawe, 2000; Wampold und Imel, 2015]. Mittlerweile existieren zur therapeutischen Beziehung und ihrer Gestaltung umfangreiche Handbücher [z. B. Hermer und Röhrle, 2008; Fiedler, 2018; Norcross und Lambert, 2019].

In der Verhaltenstherapie (VT) bzw. der kognitiven Verhaltenstherapie (KVT) hat sich das Bewusstsein für die Bedeutung der therapeutischen Beziehung durch die Entwicklungen der letzten zwei Jahrzehnte nochmals deutlich geschärft. Das Anliegen des vorliegenden Überblickartikels ist daher als Versuch einer Verortung dieser Entwicklungen im Sinne einer differentiellen Beziehungsgestaltung zu verstehen. Als Ausgangspunkt werden hierfür folgende Ansatzpunkte zur Gestaltung der Beziehung gewählt: (1) Therapiephasen, (2) Bedürfnisse, Motive und Erwartungen sowie (3) frühe traumatisierende Beziehungserfahrungen bzw. Kindesmisshandlung. Die vorgestellten Konzepte zur Beziehungsgestaltung können dabei keinerlei Anspruch auf Vollständigkeit erheben. Sie bilden in erster Linie die Landschaft der gegenwärtig der VT zugerechneten und in der VT-Ausbildung im deutschsprachigen Raum gelehrten Beziehungsgestaltungskonzepte $\mathrm{ab}$. Darauf aufbauend werden offene Fragen zur Indikation, Evidenz und praktischen Umsetzung einer differentiellen Beziehungsgestaltung diskutiert.

\section{Historische Perspektive}

Die Idee, dass die therapeutische Beziehung einen sogenannten unspezifischen Wirkfaktor ("common factor") in der Psychotherapie darstellt, hat vor allem in der von Carl Rogers [1959] begründeten Gesprächspsychotherapie eine lange Tradition. Im humanistischRogers'schen Sinne wird angestrebt, eine "echte Begegnung" in der Therapie entstehen zu lassen, womit die authentische Beziehung zwischen zwei Menschen im Mittelpunkt steht. Dieser echten Begegnung wird dabei eine heilende Wirkung zugeschrieben [Rogers, 1959]. Sie wird durch die therapeutischen Basisvariablen Echtheit oder Kongruenz des Therapeuten, Wertschätzung oder bedingungsfreies Akzeptieren und Empathie (präzises einfühlendes Verstehen) realisiert.

Demgegenüber betont die psychodynamische Tradition durch Konzepte wie Übertragung und Gegenübertragung eher die spezifische Bedeutung der therapeutischen Beziehung ("specific factor").

In der VT ist dies jedoch eine relativ neue Entwicklung: Insbesondere in der klassischen VT spielte die therapeutische Beziehung nur eine untergeordnete Rolle und wurde teilweise komplett negiert [Wolpe, 1954]. Vielmehr wurde den spezifischen Techniken und deren zugrundeliegenden Lernprozessen eine größere Bedeutung beigemessen als der direkten Arbeit an der therapeutischen Beziehung oder der Berücksichtigung früherer Beziehungserfahrungen [Bandura, 1969]. Besonders Albert Ellis lehnte die Bedeutung von Empathie und Validierung für den Therapieerfolg ab. Er relativierte jedoch später seine Auffassung, indem er eine bedingungslos akzeptierende Grundhaltung des Therapeuten gegenüber seinem Patienten als erstrebenswert formulierte [Ellis und Hoellen, 1997]. Allerdings unterstrichen bereits in den 1970er Jahren Goldfried und Davison [1976, S. 55] deutlich die Bedeutung der Beziehung für den therapeutischen Prozess: "Wer als Verhaltenstherapeut immer noch glaubt, dass Prinzipien des Lernens und der sozialen Einflussnahme ausreichten, um Verhaltensänderungen zu erreichen, hat keinen Kontakt mit der klinischen Realität" [dt. nach Zimmer, 1983a]. Auch für Aaron Beck stellte die therapeutische Beziehung bereits früh eine primäre Komponente einer effektiven Psychotherapie dar, wobei er diese allein nicht als ausreichend für den Thera- 
pieerfolg ansah [Beck et al., 1979]. Für die Realisierung des Beziehungsangebots durch den Therapeuten verwies er auf die von Carl Rogers [1959] formulierten Basisvariablen. Gleichzeitig skizzierte bereits Beck die Idee, dass die therapeutische Beziehung gezielt genutzt werden könnte, indem interpersonelle Prägungen im Rahmen der therapeutischen Beziehung bearbeitet werden, um den Patienten auf Interaktionen außerhalb der Therapie vorzubereiten.

Dementsprechend waren auch Verhaltenstherapeuten in der klinischen Realität bereits frühzeitig um einen empathisch validierenden, positiven Kontakt zu ihren Patienten bemüht [Cohen, 1984], wenngleich ein theoretisches Konstrukt geschweige denn spezifische Konzepte zur therapeutischen Beziehungsgestaltung lange nicht explizit formuliert wurden und eine empirische Untersuchung verhältnismäßig lange auf sich warten ließ.

\section{Komponenten der therapeutischen Beziehungsgestaltung}

Angestoßen wurde die Formulierung einer Definition sowie Operationalisierung der therapeutischen Beziehung in erster Linie von ausgebildeten Psychoanalytikern [Greenson, 1965; Luborsky, 1976; Bordin, 1979]. Diese Definitionen waren zunächst eng mit dem Konstrukt der Übertragung verknüpft, wobei Greenson [1965] als erster zwischen "transference" (Übertragung) und "working alliance" (Arbeitsbündnis) als verschiedene Komponenten der therapeutischen Beziehung differenzierte. Die Loslösung von der Übertragung erfolgte durch die integrativ angelegten Konzeptualisierungen der "helping alliance" durch Lester Luborsky [1976] und der "working alliance" durch Edward Bordin [1979]. Diese pantheoretischen Allianz-Konzepte stellen die Zusammenarbeit der beteiligten Akteure in den Mittelpunkt [vgl. auch Flückiger, 2018]. Bordin unterscheidet in seinem Modell drei Merkmale: (1) die Übereinstimmung in Zielen zwischen Patient und Therapeut (GOAL), (2) die Übereinstimmung in den Strategien/Techniken zur Erreichung dieser Ziele (TASK) und (3) eine affektive Beziehung (BOND).

Dieses integrative Modell, welches in Konsensus-Sitzungen der Society of Psychotherapy Research mit Vertretern diverser Therapietraditionen ausführlich diskutiert wurde [u. a. Horvath und Greenberg, 1994], bildet die Grundlage unseres heutigen Verständnisses der therapeutischen Beziehung. Zur Popularität dieses Modells trägt vor allem die offene Formulierung bei: Bordin grenzt das Arbeitsbündnis zwar von der Übertragung ab, lässt die Grenze zu anderen Konstrukten (wie z. B. Empathie, Validierung) jedoch offen, wodurch das Konstrukt keinen Anspruch darauf erhebt zu beschreiben, wie dieses Bündnis realisiert werden kann oder wodurch dieses
Bündnis aufrechtzuerhalten wäre. Allerdings betont Bordin den fortlaufenden und prozesshaften Charakter seines Konstrukts, indem er die Entwicklung der Allianz und ihr Management (Reparatur und Wiederaufbau) durch die unvermeidlichen Belastungen und möglichen Brüche im Verlauf einer Therapie als therapeutische Kernprozesse der Therapie identifiziert [Horvath, 2018]. Die Realisierung dieses Managements stützt sich dabei immer auch auf die Überprüfung der vereinbarten Ziele und Aufgaben zu deren Erreichung, womit Entwicklung und Management der Allianz immer auch spezifische Bestandteile enthalten und nicht als komplett unabhängig von den behandlungsspezifischen Strategien gesehen werden können. Dieser Aspekt entspricht einem bidirektionalen Zusammenhang im Sinne des dualen Therapiemodells [Schulte und Eifert, 2002] zwischen therapeutischer Beziehung und der (erfolgreichen) Durchführung spezifischer Techniken: Eine gute therapeutische Beziehung erleichtert die Durchführung spezifischer Techniken, wobei das Gelingen spezifischer Techniken wiederum die Beziehung verbessert [Falkenström et al., 2013]. Gleichzeitig ist eine affektive Beziehung zwischen Patient und Therapeut (BOND) neben der Zusammenarbeit bzw. Kooperation (GOALS \& TASKS) ein wichtiges Merkmal des therapeutischen Arbeitsbündnisses nach Bordin. Diese von Vertrauen und Bindung geprägte Beziehung basiert auf der authentischen Begegnung zweier Menschen und führt dazu, dass sich der Patient aufgehoben und sicher fühlen kann. Diese affektive Verbindung ist besonders dann wichtig, wenn es z. B. im Verlauf einer Therapie um schambesetzte Themen oder um das Erleben vermiedener Emotionen im Zusammenhang mit der Aufgabe von dysfunktionalen Bewältigungsstrategien geht [Lammers, 2017].

Die therapeutische Beziehungsgestaltung zielt somit auf den Aufbau und das Management eines Bündnisses zwischen Patient und Therapeut ab. Dieses auch als therapeutische Allianz bezeichnete Bündnis subsummiert neben der Zusammenarbeit auch eine affektive Beziehung und erfordert Interaktion. Diese Interaktion wird von den individuellen Patienten- und Therapeutencharakteristika gleichermaßen determiniert.

Zilcha-Mano [2017] schlägt im Zusammenhang mit den interaktionellen Patientencharakteristika ein Zweikomponentenmodell der therapeutischen Allianz vor: Demnach lässt sich die therapeutische Allianz in eine trait-Komponente (grundsätzliche Tendenz eines Patienten, befriedigende Beziehungen mit anderen aufzubauen) und eine state-Komponente (beschreibt den Prozess, wie sich Veränderungen in solchen Tendenzen durch Interaktion mit dem Therapeuten entwickeln) unterscheiden. Die trait-Komponente der Allianz spiegelt einen indirekten Zusammenhang in Bezug auf den Therapieerfolg wider, indem sie z. B. bestimmten Techniken ermöglicht, 
eine signifikante Veränderung zu bewirken. Dagegen wird der state-Komponente ein direkter Einfluss auf das Therapieergebnis zugeschrieben. Die state-Komponente bildet die Entwicklung von Fähigkeiten des Patienten ab, eine belastbare und befriedigende Allianz mit dem Therapeuten zu bilden. Verbessern sich diese Fähigkeiten, beeinflusst dies auch die allgemeinen Fähigkeiten für den Aufbau von positiven Beziehungen außerhalb der Therapie, was wiederum zu einer Symptomreduktion führt. Dies impliziert, dass der Therapeut intensiver auf die Allianz fokussieren sollte, je ausgeprägter die interaktionellen Schwierigkeiten eines Patienten sind. Das Behandlungsrational sollte demzufolge auf eine Verbesserung der interaktionellen Fähigkeiten ausgerichtet sein.

\section{Aktueller Forschungsstand}

Zur Rolle der therapeutischen Allianz liegen zahlreiche Studien vor, die für Face-to-face-Therapien in äußerst detaillierten Metaanalysen zusammengefasst wurden [u. a. Del Re et al., 2012; Flückiger et al., 2012a, b, 2013, 2018]. Darüber hinaus existieren, in bescheidenerem Umfang, metaanalytische Befunde zur onlinebasierten Therapie [Flückiger et al., 2018; Probst et al., 2019]. Flückiger et al. [2018] konnten für ihre Metaanalyse zum Zusammenhang von therapeutischer Allianz und Therapieerfolg insgesamt 295 Studien mit einer Gesamtstichprobe von über 30'000 Patienten einschließen. Dabei identifizierten sie 39 verschiedene Fragebögen, die zur Erfassung der therapeutischen Allianz eingesetzt wurden. In 150 der eingeschlossenen Studien wurde dabei das Working Alliance Inventory [Horvath und Greenberg, 1989] verwendet, welches auf Grundlage des pantheoretischen Modells von Bordin entwickelt wurde. Trotz der großen Heterogenität hinsichtlich der inhaltlichen Definition und das Operationalisieren in den einzelnen Studien unterstreichen die Ergebnisse von Flückiger et al. [2018] die Bedeutung der therapeutischen Allianz für das Gelingen einer jeden Psychotherapie. Über alle eingeschlossenen Studien hinweg findet sich eine moderate Korrelation von $r=0,278$ zwischen Behandlungserfolg und therapeutischer Allianz. Diese Korrelation entspricht einer mittleren Effektstärke (Cohens $d=0,58)$, was verglichen mit der ebenfalls gut beforschten Protokolladhärenz $(d=0,04)$ oder der Kompetenz des Therapeuten $(d=0,14)$ eine besondere Bedeutung der therapeutischen Beziehung nahelegt [Wampold et al., 2018, S. 324]. Diese Gegenüberstellung soll an dieser Stelle jedoch keine Trennung zwischen den einzelnen Wirkfaktoren implizieren, da sich auch empirisch Hinweise auf positive Wechselwirkungen zwischen spezifischen Techniken und der therapeutischen Beziehung finden lassen [Falkenström et al., 2013].
Neben der therapeutischen Allianz sind einzelne Elemente der therapeutischen Beziehung im Hinblick auf ihren Zusammenhang mit dem Therapieerfolg Gegenstand der Forschung. In ihrer Zusammenstellung der empirischen Befunde zu diesen Elementen beziehen Norcross und Lambert [2018] allgemeingültig angelegte Gesamtkonzepte (therapeutische Allianz, "real relationship") und Teilkomponenten ("goal consensus, collaboration") sowie eher gestalterische Konstrukte (Selbstöffnung), welche im deutschsprachigen Raum teilweise als schulenspezifisch eingeordnet werden (Umgang mit Gegenübertragung), mit ein. Gleichzeitig wurden Variablen identifiziert, für die sich eine Anpassung der Beziehungsgestaltung als günstig erwiesen hat. Bei der empirischen Einordnung dieser Elemente entschied ein Expertengremium bestehend aus 10 Mitgliedern unabhängig voneinander über den Grad der empirischen Evidenz in Bezug auf den Therapieerfolg anhand standardisierter Kriterien (Tab. 1).

Norcross und Lambert [2018] betonen für diese Zusammenstellung eindringlich, dass die separate Präsentation der einzelnen Elemente zu kurz greift und diese teilweise stark untereinander korrelieren. Auch lassen sich keine Aussagen darüber treffen, wann welche Beziehungselemente für welchen Patienten in welcher Situation besonders wertvoll sind. Dies obliegt allein der Entscheidung des jeweiligen Therapeuten. Berücksichtigt man an dieser Stelle, dass Therapeuteneffekte bis zu 8\% der Varianz im Therapieergebnis erklären [Barkham et al., 2017], liegt die Vermutung eines Zusammenhangs mit der Qualität der therapeutischen Allianz nahe. $\mathrm{Zu}$ dieser Frage konnten Del Re et al. [2012] in ihrer Metaanalyse zeigen, dass Therapeuten, die im Mittel eine qualitativ stärkere therapeutische Allianz mit ihren Patienten aufbauen konnten, insgesamt auch bessere Therapieergebnisse erzielten. Allerdings bleibt offen, was genau diese Therapeuten im Vergleich zu ihren Kollegen, auch in Bezug auf die individuelle Beziehungsgestaltung, auszeichnet.

\section{Neuere Beziehungsgestaltungskonzepte in der VT im deutschsprachigen Raum}

Für die VT formulierten Zimmer und Zimmer [1996a] im deutschsprachigen Raum das Konzept der funktionalen Beziehungsgestaltung. Demnach gibt es in der VT keine verbindliche einheitliche Konzeptualisierung der therapeutischen Beziehungsgestaltung. Vielmehr zeigt sich die funktionale Beziehungsgestaltung in den unterschiedlichen Verhaltensweisen des Therapeuten in Bezug auf einen bestimmten Patienten mit seinen individuellen Bedürfnissen, Motiven, Erwartungen und Problemen bzw. negativen Erfahrungen, seinem Umgang mit Emo- 
Tabelle 1. Zusammenfassung metaanalytischer Zusammenhänge zwischen Elementen der therapeutischen Beziehung und dem Behandlungsergebnis nach Norcross und Lambert [2018]

\begin{tabular}{llrr}
\hline Evidenzstärke & Konzepte/Elemente der therapeutischen Beziehung & Studienanzahl (N) & $\begin{array}{l}\text { Korrelation } \\
(\text { Effektstärke })^{\mathrm{a}}\end{array}$ \\
\hline Nachweisbar effektiv & Allianz in der individuellen Psychotherapie & $306(30,000+)$ & $0.28(0.57)$ \\
& Zusammenarbeit (Kollaboration) & $53(5,286)$ & $0.29(0.61)$ \\
& Übereinstimmung in den Therapiezielen & $54(7,278)$ & $0.24(0.49)$ \\
& Kohäsion & $55(6,055)$ & $0.26(0.56)$ \\
& Empathie & $82(6,138)$ & $0.28(0.58)$ \\
& Positive Wertschätzung und Anerkennung & $64(3,528)$ & $-(0.28)$ \\
& Feedback & $24(10,921)$ & $-(0.14-0.49)^{\mathrm{b}}$ \\
\hline Möglicherweise effektiv & Kongruenz/Echtheit & $21(1,192)$ & $0.23(0.46)$ \\
& "Echte Beziehung" (real relationship) & $17(1,502)$ & $0.37(0.80)$ \\
& Ausdruck von Emotionen & $42(925)$ & $0.40(0.85)$ \\
& Förderung und Entwicklung von positiven Erwartungen & $81(12,722)$ & $0.18(0.36)$ \\
& Förderung der Glaubwürdigkeit der Behandlung & $24(1,504)$ & $0.12(0.24)$ \\
& Umgang mit Gegenübertragung & $9(392$ Therapeuten $)$ & $0.39(0.84)$ \\
& Reparieren von Allianzbrüchen & $11(1318)$ & $0.30(0.62)$ \\
\hline Vielversprechend, aber & Selbstöffnung und Beziehungsklären & $21(\sim 140)$ & NA \\
\hline unzureichende Forschung & & & \\
\hline
\end{tabular}

${ }^{a}$ Cohens $d$ oder Hedges $g .{ }^{\mathrm{b}}$ In Abhängigkeit von der Vergleichsgruppe und der Feedbackmethode (Feedback erscheint effektiver bei Patienten mit einem erhöhten Risiko für eine Verschlechterung der Symptomatik und weniger effektiv für alle Patienten).

Abb. 1. Ausrichtung der Beziehungsgestaltung im Rahmen einer differentiellen Beziehungsgestaltung.

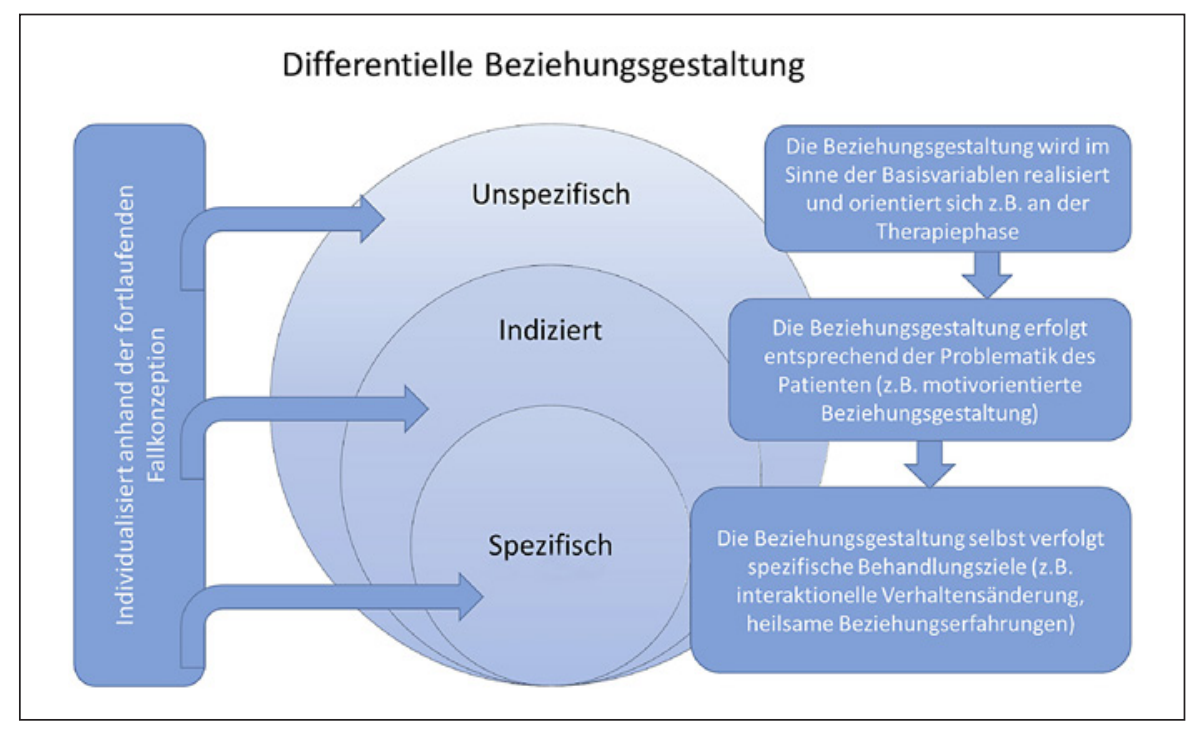

tionen zu einem ganz bestimmten Zeitpunkt oder einer Situation im therapeutischen Prozess. Dementsprechend sieht auch Lammers [2017] den Versuch, die ideale therapeutische Beziehungsgestaltung mit dem Ziel zu charakterisieren, diese methodisch mit allen Patienten realisieren zu können, als weder hilfreich noch praktikabel an. Allerdings existieren mittlerweile in der VT und insbesondere den Ansätzen der "Dritten Welle" verschiedene teilweise sehr spezifisch ausformulierte Strategien, welche sich in der Beziehungsgestaltung konzeptuell unter- scheiden und unterschiedliche Schwerpunkte setzen. Gemeinsam ist den verschiedenen Strategien dabei, dass die therapeutische Beziehung als weit mehr als nur ein "Arbeitsbündnis" interpretiert wird. Der Therapeut nutzt die Gestaltung oder die Dynamik der Beziehung im Sinne eines strukturierten und operationalisierten Behandlungselementes spezifisch und bewusst zur Veränderung von Erwartungshaltungen sowie in Abhängigkeit der Problemlage. Gleichzeitig wird die therapeutische Beziehung in vielen Ansätzen auch als Plattform der Mani- 
festation von Schemata, Erwartungen und Verhaltensmustern im Rahmen des biographischen Kontexts angesehen. Diese spezifischen Ansätze sollten in einer differentiellen Beziehungsgestaltung explizit berücksichtigt werden. Die Art und Weise bzw. der Fokus in der Beziehungsgestaltung wird dabei im Sinne der gemeinsamen Therapieziele entsprechend dem größtmöglichen evidenzbasierten Nutzen für den individuellen Patienten ausgewählt und aufgebaut (Abb. 1). Eine differentielle Beziehungsgestaltung kann demnach als die evidenzbasierte Adaptation der Beziehungsgestaltung vor dem Hintergrund einer fortlaufenden individualisierten Fallkonzeption verstanden werden. Dies entspricht im Wesentlichen einer differentiellen Therapieplanung auf der Basis eines integrativen Störungsmodells [Rief und Strauß, 2018].

Im Folgenden sollen verschiedene verhaltenstherapeutische Ansätze vorgestellt werden, welche über die "working alliance" hinaus in der VT bzw. der VT zugeordneten Ansätzen Anwendung finden und sich bei der Gestaltung der therapeutischen Beziehung an unterschiedlichen Aspekten bzw. Ansatzpunkten orientieren. Folgende Ansatzpunkte der Beziehungsgestaltung können unterschieden werden:

(a) Therapiephasen;

(b) Bedürfnisse, Motive und Erwartungen des Patienten;

(c) Kindesmisshandlung [childhood maltreatment; World Health Organization, 2016] in Verbindung mit heutigen interpersonellen Problemen.

Basierend auf dieser Einteilung werden die verschiedenen Strategien im Folgenden beschrieben.

\section{Therapiephasen als Ansatzpunkt der \\ Beziehungsgestaltung}

Für konzeptuelle Überlegungen zur differentiellen Gestaltung der therapeutischen Beziehung lohnt sich eine Orientierung an Prozessmodellen der Psychotherapie [z. B. das 7-Phasen-Modell von Kanfer et al., 2012]. Den einzelnen Phasen im therapeutischen Prozess lassen sich dabei spezifische therapeutische Aufgaben zuordnen, wobei die Beziehungsgestaltung durch den Therapeuten als fortwährende aktive Aufgabe betont wird [u. a. Schulte, 1996]. Für die Arbeit an der Beziehung werden in Prozessmodellen zumeist drei Hauptphasen differenziert: die Eingangs- bzw. Initialphase, die Haupt- oder Behandlungsphase sowie die Abschluss- bzw. Abschiedsphase [vgl. Lairaiter, 2008; Lammers, 2017]. Ein spezifisches Verfahren, welches sich in der Gestaltung der Beziehung explizit an der jeweiligen Behandlungsphase orientiert, ist die Interpersonelle Psychotherapie (IPT) [Weissman et al., 2000; dt. Schramm, 2019; für einen Überblick Brakemeier und Frase, 2012], auf welche im Folgenden daher immer wieder verwiesen wird.
Die Eingangs- bzw. Initialphase der Therapie

Für diese erste Therapiephase ist die Herstellung einer positiven, vertrauensvollen und tragfähigen Arbeitsbeziehung vorrangige Aufgabe des Therapeuten. Die praktische Umsetzung dieser so einfach anmutenden wie offensichtlichen Beziehungsaufgabe erfordert dabei jedoch immer eine individuelle Adaptation des therapeutischen Beziehungsangebotes durch den Therapeuten an den individuellen Patienten. In erster Linie sollten im Rahmen dieser Eingangsphase in der VT - abgesehen von der Herstellung der Arbeitsbeziehung - vor allem organisatorische und diagnostische Aufgaben adressiert werden. Die Vorgabe einer Struktur ermöglicht es dem Patienten, sich seinerseits ein Bild vom Therapeuten und den Kontextfaktoren (Setting, Frequenz, etc.) zu machen und eine Idee davon zu bekommen, wie die gemeinsame Arbeit im Rahmen einer Psychotherapie aussehen kann. Dies ermöglicht dem Patienten, sein individuelles Beziehungsangebot an den Therapeuten entsprechend anzupassen und gegebenenfalls zu intensivieren. Die Beziehungsgestaltung durch den Therapeuten in der IPT entspricht dabei der eines sogenannten Advokaten, der sich als ermutigender, wohlwollender, optimistischer und unterstützender "Anwalt" für seinen Patienten versteht. Besonders in der Frühphase nimmt der Therapeut dabei eine aktive und direktive Grundhaltung ein. Er tritt explizit als Experte auf, erläutert die strukturierten Bausteine und vermittelt Hoffnung, indem er positive Erwartungen schürt. Dem Patienten wird dabei die Notwendigkeit einer "aktiven Krankenrolle" vermittelt (Betonung der Bedeutung, aktiv an der Therapie mitzuwirken bei gleichzeitiger Anerkennung der psychischen Störung als Krankheit).

Die Haupt- bzw. Behandlungsphase der Therapie

In der Hauptphase der Therapie liegt der Fokus auf konkreten Interventionen. Hierbei werden die Bewertungen und Verhaltensweisen des Patienten mit dem Ziel der Veränderung hinterfragt, was ein verstärktes Arbeiten auf Patientenseite notwendig macht. Durch das eher anleitend-strukturierte und zeitgleich zunehmend passivere bzw. Verantwortung delegierende Verhalten des Therapeuten entsteht somit der notwendige Veränderungsdruck beim Patienten, welcher in dieser Therapiephase immer wieder spürbar werden sollte [Lammers, 2017]. Die Gestaltung der therapeutischen Beziehung in dieser Phase wurde von Zimmer [1983b] als ein Kontinuum beschrieben, dessen Pole Unterstützung, Förderung, Verständnis und Wärme auf der einen Seite sowie Forderung, Kontrolle, Eigenverantwortung und Konfrontation auf der anderen Seite beinhalten. Der Therapeut sollte sein Verhalten auf diesem Kontinuum flexibel, entsprechend den jeweiligen Rahmenbedingungen und Problemen bzw. Bedürfnissen des Patienten, ausrichten [vgl. Zimmer, 1983b]. Nach Lairaiter [2008] können die je- 
weils eingesetzten Interventionen mit unterschiedlichen Beziehungsqualitäten assoziiert sein. Zum Beispiel erfordern konfrontative Methoden ein starkes Vertrauensverhältnis [Scholz, 1998], wohingegen der Aufbau von positiven Aktivitäten eher mit einer ressourcenorientierten, aber auch pädagogischen Arbeitshaltung assoziiert ist. Auch in der IPT nimmt der Therapeut sich im Verlauf mehr und mehr zurück, um dem Patienten Raum zu geben, sich aktiver einzubringen. Der Therapeut verhält sich dementsprechend passiver bzw. abwartender als in der Initialphase, verlässt dabei jedoch nie die Rolle des unterstützenden Advokaten [Schneibel et al., 2017].

Die Abschluss- bzw. Abschiedsphase der Therapie

Wenn die wichtigsten Therapieziele erreicht wurden, beginnt die Abschlussphase der Therapie. In dieser übernimmt der Patient im Idealfall immer mehr Eigenverantwortung für sein Verhalten und setzt hilfreiche Strategien im Sinne des Selbstmanagements bzw. der Hilfe zur Selbsthilfe (self-therapy) eigenständig um. Im Vordergrund stehen dabei die Stabilisierung und der Transfer des bisher Erreichten. In der Vorbereitung der bevorstehenden Beendigung der Therapie und der damit verbundenen Ablösung sollte sich der Therapeut zunehmend aus seiner formalen Verantwortung zurückziehen, um dem Patienten gleichermaßen zunehmend Autonomie zukommen zu lassen [Kanfer et al., 2012]. Insbesondere die Ablösung und das damit verbundene Beenden der therapeutischen Beziehung sind oftmals mit hohen Anforderungen an die therapeutische Beziehungsgestaltung verbunden [Lairaiter, 2008]. In der IPT werden in der Abschlussphase ebenfalls die Stabilisierung und die Eigenverantwortung des Patienten betont. Der Therapeut verstärkt dabei die Erfolge des Patienten. Die Rolle des Advokaten verlässt der Therapeut auch in dieser Phase nicht, da er dem Patienten auch in der Abschlussphase der Therapie weiterhin transparent und stützend zur Verfügung steht. Dabei steht die Thematisierung der interpersonellen Besonderheiten des Abschieds und damit verbundener Emotionen explizit im Fokus, wodurch ein verabschiedendes Gestalten der Beziehung durch den Therapeuten ermöglicht wird.

\section{Bedürfnisse, Motive und Erwartungen des Patienten} als Ansatzpunkt der Beziehungsgestaltung

Eine Ausrichtung der Beziehungsgestaltung durch den Therapeuten an den individuellen Bedürfnissen, Motiven und Erwartungen des Patienten bedingt notwendigerweise, dass der Therapeut diese auch kennt. Um dies zu gewährleisten, haben Klaus Grawe und Franz Caspar eine vertikale Verhaltensanalyse zum Problemverhalten von Patienten - die Plananalyse - entwickelt [Caspar, 2018]. Die Plananalyse erfasst die hinter einem Verhalten stehende Motivstruktur, wobei sich die Strategien (Pläne) zur Erreichung der verhaltenssteuernden Motive und Bedürfnisse in den sozialen Interaktionen des Individuums wiederfinden. In der Beziehungsgestaltung orientiert sich der Therapeut an diesen übergeordneten Motiven (motivorientierte Beziehungsgestaltung) und verhält sich komplementär zu diesen. Komplementarität bedeutet, dass der Therapeut zunächst danach fragt, welchem Zweck oder Ziel ein bestimmtes interpersonelles oder intrapsychisches Verhalten des Patienten dient, und anschließend seine Beziehungsgestaltung dementsprechend ausrichten kann. Dem Patienten wird es somit ermöglicht, sich einerseits in seinen bewussten Veränderungszielen unterstützt zu wissen, und andererseits den Weg für die therapeutische Veränderung durch die Sättigung therapiebehindernder Motive frei zu machen. Darüber hinaus soll sich der Patient auch als Mensch im Kontext der therapeutischen Beziehung gesehen und unterstützt fühlen, wodurch die Beziehung im Sinne einer korrigierenden Erfahrung nutzbar gemacht wird [Caspar und Belz, 2017]. Dabei entspricht die Fähigkeit des Therapeuten, sich den wichtigsten Bedürfnissen des Patienten gegenüber komplementär zu verhalten, einer ressourcenorientierten Haltung [vgl. Lammers, 2017]. Die Ressourcenperspektive fokussiert dabei aktiv auf die Suche und Nutzbarmachung von positiven und hilfreichen Aspekten, wobei die individuellen Fähigkeiten, Interessen und Erfolge des Patienten explizit thematisiert werden. Diese ressourcenorientierte Beziehungsgestaltung [Grawe, 2000] ebnet dem Patienten das Erleben von positiven Emotionen, indem eigene Kompetenzen im Blickfeld erscheinen [Flückiger und Holtforth, 2007; Scheel et al., 2013].

Im Rahmen der für Patienten mit Persönlichkeitsstörungen entwickelten klärungsorientierten Psychotherapie von Rainer Sachse [1997; Sachse et al., 2011] findet das Konzept der komplementären Beziehungsgestaltung im Hinblick auf die jeweils spezifische Pathologie der einzelnen Persönlichkeitsstörungen eine speziell für diese Patientengruppe ausformulierte praktische Anwendung. Besonders herausgestellt wird dabei die Unterscheidung zwischen impliziten und expliziten Motiven. Sachse [1997] betont in seinem Modell der doppelten Handlungsreaktion ebenfalls eine zugrundeliegende implizite Motivebene, im Rahmen derer die Erfüllung interaktioneller Motive, wie beispielsweise Anerkennung oder Autonomie, einen zentralen Stellenwert hat. Auf einer zweiten Ebene bilden sich infolge von interpersonellen Frustrationserfahrungen bei der authentischen Motivbefriedigung negative kognitiv-affektive Schemata heraus. Im Zusammenhang mit diesen dysfunktionalen Schemata stehen vor allem negative Beziehungserwartungen, aufgrund derer sich Patienten auf einer dritten Ebene (Spielebene) unmittelbar sicht- und erlebbar verhalten. Auf der Spielebene versuchen Patienten durch strategische Verhaltensweisen zumindest eine teilweise Befrie- 
digung der frustrierten authentischen Motive zu erzwingen.

Besonders zu Beginn der therapeutischen Arbeit ist es deshalb essentiell, sich als Therapeut - genauso wie in der motivorientierten Beziehungsgestaltung - komplementär zu den authentischen Motiven des Patienten zu verhalten, um zugrundeliegende Bedürfnisse zu sättigen. Gleichzeitig soll dadurch im Beziehungsangebot eine Aktivierung negativer Schemata vermieden werden. Durch diese Art der Beziehungsgestaltung gelingt es dem Therapeuten, einen ausreichenden Beziehungskredit beim Patienten aufzubauen, was in der Folge die weiterführende Bearbeitung der Schemata ermöglicht. Dementsprechend betont Sachse [2006], dass die Beziehungsgestaltung als die eigentliche Basis von Psychotherapie fungiert und in jeder eingesetzten Intervention im gesamten Therapieverlauf ständig realisiert werden sollte, da individuell und punktuell resultierende korrigierende Beziehungserfahrungen durch die komplementäre Beziehungsgestaltung allein nicht ausreichend sind, um dysfunktionale Schemata dauerhaft zu verändern.

In diesem Zusammenhang wurde auch die Rolle von Patientenerwartungen als aufrechterhaltende Bedingung für die Symptomatik im Kontext von integrativen Störungsmodellen zur Therapieplanung herausgestellt [Rief und Strauß, 2018]. Hinsichtlich der Optimierung psychotherapeutischer Interventionen besteht deshalb die Möglichkeit, besonders auf Patientenerwartungen zu fokussieren. Hierzu haben Rief und Glombiewski [2016] den Ansatz der erwartungsfokussierten psychotherapeutischen Interventionen formuliert. Relevante Patientenerwartungen können dabei direkt in Verbindung mit dem Behandlungserfolg, der Störung und störungsrelevanten Situationen sowie der eigenen Person und anderen stehen (z. B. Erwartungen an die Unterstützung durch den Therapeuten). Da Patientenerwartungen bezüglich des Behandlungsergebnisses zu den stärksten Prädiktoren für den Erfolg unterschiedlicher medizinischer Behandlungen zählen, sollten psychologische Interventionen der Beziehungsgestaltung dementsprechend auf die Erfassung und Optimierung von Patientenerwartungen abzielen [Kube et al., 2019]. Sind dem Therapeuten diese Erwartungen bekannt, kann er seine Beziehungsgestaltung entsprechend ausrichten. Dabei können positive Beziehungserwartungen verstärkt bzw. stabilisiert werden. Demgegenüber kann der Therapeut durch ein konträres Verhalten zu negativen Beziehungserwartungen des Patienten Veränderungsprozesse in Bezug auf generalisierte Beziehungserwartungen anstoßen. $\mathrm{Zu}$ beachten ist jedoch, dass Patienten vor allem durch Prozesse der selektiven Aufmerksamkeit erwartungskorrigierende Erfahrungen in ihrer individuellen Bewertung vernachlässigen bzw. nicht automatisch berücksichtigen. Aus diesem Grund ist es essentiell, sich im Zuge erwartungsfokussier- ter Interventionen speziell mit den individuellen Mechanismen auseinanderzusetzen, die zur Persistenz der Erwartungen führen.

Nicht zuletzt sind Patientenerwartungen auch für die Qualität der therapeutischen Beziehung relevant [Fuertes et al., 2015]. Dabei scheint bereits die Erwartung, dass die Patientenmeinung im Zuge einer psychotherapeutischen Behandlung wertgeschätzt wird, die Qualität der therapeutischen Allianz zu verbessern [Flückiger et al., 2012b]. Im Prozess kann es vor allem für Patienten mit (frühen) traumatisierenden Beziehungserfahrungen (Kindesmisshandlung) hilfreich sein, individuelle Beziehungserwartungen des Patienten an den Therapeuten gemeinsam zu erarbeiten und zu überprüfen. Somit könnte die Gestaltung der Beziehung im Hinblick auf spezifische Behandlungsziele auch als erwartungskorrigierende Intervention konzeptualisiert werden [Rief und Strauß, 2018], was im folgenden Absatz näher erläutert wird.

\section{Kindesmisshandlung in Verbindung mit heutigen interpersonellen Problemen}

Eine weitere Möglichkeit der therapeutischen Beziehungsgestaltung ist somit die Orientierung an spezifischen interpersonellen Problemen des Patienten unter Berücksichtigung biographischer Lernerfahrungen, insbesondere von Kindesmisshandlungen (Vernachlässigung und/oder Missbrauch). Vor allem die dialektischbehaviorale Therapie (DBT), das Cognitive Behavioral Analysis System of Psychotherapy (CBASP) und die Schematherapie, welche auch als sogenannte "DritteWelle Verfahren der VT" bezeichnet werden, stellen spezifische Strategien der Beziehungsgestaltung zur Verfügung. Sie unterscheiden sich jedoch zum Teil deutlich in der Anwendung von Techniken und Haltungen, was im Folgenden dargestellt werden soll. Beispiele zur praxisnahen Umsetzung der einzelnen Ansätze finden sich u. a. in einem Buchkapitel von Brakemeier et al. [2017] sowie einer Video-Learning DVD von Brakemeier [2019].

\section{Beziehungsgestaltung in der Dialektisch-}

Behavioralen Therapie (DBT)

Die DBT nach Marsha Linehan stellt eine störungsspezifische Therapie der emotional-instabilen Persönlichkeitsstörung vom Borderline-Typ (BPS) dar [Linehan, 1993]. Nach Linehan steht bei der BPS die Störung der Emotionsregulation im Vordergrund. Gleichzeitig können neurobiologische Vulnerabilitäten, frühe traumatische Erlebnisse und ein in der frühen Entwicklungsphase invalidierendes psychosoziales Umfeld zur Ausgestaltung einer komplexen emotionalen Dysregulation mit dysfunktionalen Grundannahmen und Handlungsfolgen führen. Haupttechniken in der DBT sind neben KVTStrategien vor allem Gestalttherapie- und Meditationstechniken. Hauptziel ist dabei die Reduktion selbstschä- 
digender Verhaltensweisen, eine verbesserte Emotionsregulation und die Behandlung traumatischer Ereignisse. Ein wesentliches Element in der DBT ist die sogenannte dialektische Beziehungsgestaltung [Linehan, 1993; Barnicot et al., 2012; Linehan, 1993]. Die Grundannahme ist hierbei, dass aus der Entwicklung von Widersprüchen neue Sichtweisen und Haltungen entstehen können. Die therapeutische Rolle versteht sich in der Begleitung des schmerzhaften Prozesses aus Veränderung und Akzep$\tan z$ und ist vergleichbar mit der eines Trainers (Cheerleading). Auf dem Weg zur Synthese kann es aufgrund des Ambivalenzkonflikts zu "Beziehungskämpfen" in der therapeutischen Arbeit kommen. Daher versucht der DBT-Therapeut ständig selbst “in Bewegung zu sein”, um einen Stillstand in der therapeutischen Arbeit zu vermeiden (sog. DBT-Wippe). Um ein Ungleichgewicht zu verhindern (d.h. beispielsweise auf distanziertes Verhalten des Patienten mit konsekutiver Nähe zu reagieren), versucht der DBT-Therapeut einen Ausgleich zu schaffen und nicht dem permanenten Oszillieren des BPS-Patienten hinsichtlich Nähe und Distanz zu folgen. So ist es Ziel der Beziehungsgestaltung, durch eine paradoxe Reaktion den Patienten in seiner Haltung zu irritieren und ihn zu einem spontanen Schritt in Richtung des gewünschten Zieles zu provozieren. Dabei sind vor allem die Grundansätze von Akzeptanz und Veränderung in einem polarisierenden Wechselspiel im Fokus des Therapeuten. Über die verschiedenen Formen des Validierens wird die Akzeptanz des Patienten gefördert, aber gleichzeitig u. a. über Skills an Veränderungsstrategien gearbeitet (Beziehungsorientierung im Sinne von Akzeptanz, Validierung). Dieses wird auch in der Kommunikation mit dem Patienten praktiziert. Hierbei wird in der Beziehungsgestaltung einerseits das Miteinander betont, in Form einer reziproken Kommunikation (z. B. auch in Form von Telefonanrufen durch den Patienten innerhalb bestimmter Grenzen, um eine warme und zugewandte Kommunikation $\mathrm{zu}$ realisieren). Andererseits ist es im Sinne der Dialektik auch notwendig, die eigenen therapeutischen Grenzen zu betonen. Der warmen, zugewandten Kommunikation steht hierbei auch eine konfrontative Kommunikation gegenüber, bei der es Ziel ist, einen (festgefahrenen) Affekt zu verlagern und einen anderen Standpunkt einzunehmen. Zusammenfassend ermöglicht die dialektische Beziehungsgestaltung die Chance, Veränderungsbereitschaft beim Patienten zu fördern, und adressiert dabei gleichzeitig das Grundproblem des Nähe-Distanz-Konflikts bei Patienten mit BPS.

Beziehungsgestaltung im Cognitive Analysis System of Psychotherapy (CBASP)

CBASP wurde von James P. McCullough [2000] spezifisch für die Behandlung der chronischen bzw. persistierenden Depression entwickelt. CBASP kombiniert als integrative, schulenübergreifende Therapie kognitivemotionale, verhaltensorientierte, interpersonelle und psychodynamisch-analytische Theorien und Techniken und kann am ehesten als interpersonelle Lerntherapie beschrieben werden.

Chronisch depressive Patienten haben häufig negative Beziehungserfahrungen in der Kindheit und im weiteren Leben erfahren (Kindesmisshandlung wie Vernachlässigung oder Missbrauch), welche sich bis heute in Form von interpersonellen Problemen (wie rigide oder extreme Verhaltensweisen, Empathiedefizite, schwierige Beziehungen oder Isolation) auswirken. Daher besteht ein zentrales Ziel der CBASP-Therapie darin, den Patienten durch die aktive Gestaltung der therapeutischen Beziehung heilsame korrigierende Beziehungserfahrungen zu ermöglichen [McCullough, 2006]. Patienten mit frühen traumatisierenden Beziehungserfahrungen können die Tendenz zeigen, den Therapeuten mit einer wichtigen verletzenden Bezugsperson aus ihrer Vergangenheit gleichzusetzen (Übertragung). Dadurch können Veränderungsmotivation und Verhaltensänderung behindert werden, da Patienten implizit erwarten, dass auch der Therapeut sie zurückweist, bestraft, verlässt, missbraucht, etc. Daher soll in CBASP eine sichere, authentische und menschliche Begegnung mit dem Therapeuten realisiert werden [McCullough, 2006], was dadurch erreicht wird, dass der CBASP-Therapeut sich immer wieder bewusst persönlich einbringt (kontrolliert-persönliches Einlassen, disciplined personal involvement, DPI). Diese therapeutische Haltung bedeutet, dass er seine eigenen Gefühle und Reaktionen als Konsequenz des Verhaltens des Patienten einsetzt, wobei diese Technik als kontingente persönliche Reaktion bezeichnet wird [z. B. McCullough, 2000, 2006]. Anschließend wird durch interpersonelle Diskriminationsübungen die Reaktion des Therapeuten verglichen mit Reaktionen der prägenden Bezugspersonen in ähnlichen Situationen. In der praktischen Umsetzung kann hierfür zudem auf den Kiesler-Kreis - ein interpersonelles Circumplexmodell [Kiesler, 1983] - zurückgegriffen werden, um interpersonelles Verhalten einzuordnen und komplementäres Reaktionsverhalten für den Patienten erklärbar zu machen.

Diese Art der Beziehungsgestaltung fordert folglich eine nicht-neutrale, sehr persönliche, "menschliche" Rolle des Psychotherapeuten, wobei diese nahezu im Gegensatz zu stehen scheint zum "klassischen" psychodynamischen, nicht persönlich agierenden, neutralen und abstinenten Therapeuten. McCullough postuliert, dass durch diese therapeutische Beziehungsgestaltung die durch die negative Beziehungserfahrungen entstandene Entkopplung der Wahrnehmung des Betroffenen von seiner Umwelt aufgelöst und das präoperatorische Funktionsniveau sowie die "interpersonelle Mauer" überwunden werden können [McCullough, 2003]. 
Für früh traumatisierte Patienten erscheint dieses Vorgehen aus drei Gründen relevant [nach Brakemeier et al., 2017]:

(1) Therapeuten können ihren Patienten nur dann vermitteln bzw. rückmelden, wie diese mit anderen Menschen empathisch umgehen, wenn sie selbst bereit sind, persönliche Gefühle und Reaktionen preiszugeben (Modelllernen, Empathietraining).

(2) Für viele Patienten ist eine sichere und authentische menschliche Begegnung mit ihrem Therapeuten eine neuartige Erfahrung. Wenn sich die Therapeuten so persönlich und menschlich zeigen, können die Patienten Diskriminationskriterien entdecken, um die aktuelle therapeutische Beziehung mit früheren dysfunktionalen Beziehungen zu vergleichen und Reaktionen zu unterscheiden zu lernen. Diese Entwicklung wird durch interpersonelle Diskriminationsübungen unterstützt.

(3) Schließlich kann feindselig wirkenden Patienten nur die persönliche Reaktion der Therapeuten helfen zu erkennen, dass ihr feindseliges oder destruktives Verhalten bei anderen Mitmenschen verletzende Auswirkungen hat und Distanz erzeugt, was sie häufig wegen des "Wahrnehmungsdilemmas" nicht mitbekommen.

Um diese Haltung der Beziehungsgestaltung als Therapeut zu verstehen und anzuwenden, ist es hilfreich, sich die Bedeutung jedes einzelnen Wortes des DPI zu verdeutlichen [nach Brakemeier, Guhn und Normann, 2021]:

- Diszipliniert (auch im Sinne von kontrolliert oder überlegt): Therapeuten werden angehalten, sich vor dem persönlichen Einlassen genau zu überlegen, ob, was und wie sie den Patienten Emotionen und/oder Verhaltenstendenzen mitteilen sowie welches Ziel bzw. welche heilsame Beziehungserfahrung sie dabei verfolgen.

- Persönlich: Therapeuten sind bereit, Patienten eine persönliche, authentische, offene, gefühlsmäßige Reaktion mitzuteilen; sie sollen also als "menschliche Wesen" reagieren.

- Einlassen: Die Therapeuten sind grundsätzlich bereit, sich auf den Patienten persönlich einzulassen. Die Voraussetzung dafür sollte immer sein, dass sie dem Patienten grundsätzlich wohl gesonnen sind, ihn mögen, da sie aufgrund der ihnen bekannten frühen Beziehungserfahrungen auch schwierige Verhaltensweisen einordnen und verstehen können.

Deutlich wird, dass DPI nur angewendet werden sollte, wenn der Therapeut auch Einsicht in die frühen schwierigen Beziehungserfahrungen des Patienten hat.

Beziehungsgestaltung in der Schematherapie

Neben den Elementen der KVT werden in der Schematherapie vor allem erlebnisorientierte Techniken ge- nutzt. Die ursprünglichen Konzepte gehen im Wesentlichen auf Jeffrey Young zurück [Young, 1994; Young et al., 2003] und zielen auf die Identifikation maladaptiver Schemata ab. Ein maladaptives Schema entsteht durch schädliche Kindheitserlebnisse, die auf der Verletzung menschlicher Grundbedürfnisse basieren ( $\mathrm{z}$. B. sichere Bindung, Autonomie). Hieraus können sich sogenannte maladaptive Schemata entwickeln [Young et al., 2003]. Die Realisierung einer warmherzigen stabilen Beziehung ist ein Kernelement der Schematherapie. Eine besondere Rolle spielt hierbei die "begrenzte Nachbeelterung" ("limited reparenting"), welche auf die Begrifflichkeit von Alexander und French [1946] zurückgeht. Wesentliche Aspekte sind neben Authentizität auch eine begrenzte Selbstöffnung des Therapeuten gegenüber dem Patienten. Frustrierte Grundbedürfnisse in der Kindheit sollen im Rahmen der therapeutischen Beziehung befriedigt werden, wobei zugleich auch dysfunktionale Verhaltensweisen im Rahmen einer empathischen Konfrontation angesprochen und begrenzt werden. Die begrenzte Nachbeelterung findet sowohl im Rahmen kognitiver Strategien als auch während emotionsorientierten Techniken (Imagination, Stühlearbeit, Briefe schreiben) statt [Young et al., 2003]. Auf ein frühes maladaptives Schema in der Domäne "Misstrauen/Missbrauch" würde sich der Therapeut beispielsweise explizit vertrauenswürdig und aufrichtig verhalten und den Patienten auf eventuelle negative Gefühle oder Erwartungen dem Therapeuten gegenüber ansprechen. Der Therapeut steht hierbei selbst zur eigenen Unvollkommenheit und kann persönliche Schwächen zugeben. Grundsätzlich ist in der Nachbeelterung die Selbstöffnung des Therapeuten hinsichtlich eigener Emotionen, Gedanken und Erfahrungen integraler Bestandteil. Der Beziehungsstil ist hierbei vor allem auf die frustrierten Grundbedürfnisse ausgelegt. Ziel ist es also, den Patienten aus dem Erlebens- in den Reflexionszustand zu führen, um neue Verhaltensstrategien zu erlernen. Der Therapeut dient als Modell, bis der Patient selbst einen "gesunden Erwachsenen" aufbauen kann. Dies geht soweit, dass der Therapeut im Rahmen des Imagery Rescripting beim Überschreiben der traumatischen Erinnerung selbst als Helferfigur die imaginierte Szene betritt [vgl. Jacob und Seebauer, 2013]. Diese Form der Beziehungsgestaltung ermöglicht eine sehr starke emotionsfokussierte Arbeit mit dem Ziel der Stärkung der Persönlichkeitsstruktur. Durch die begrenzte Nachbeelterung können für den Patienten schwierige emotionale Situationen durch eine Integration in das Selbstbild bearbeitet werden und zugleich funktionale Anteile wie der Modus des gesunden Erwachsenen gestärkt werden. 


\section{Auf dem Weg zu einer differentiellen und individualisierten Beziehungsgestaltung?}

Die Möglichkeiten zur Realisierung der therapeutischen Beziehungsgestaltung in der VT sind durch neuere Behandlungsansätze theoriegeleitet ausformuliert worden. Im Hinblick auf die teilweise spezifischen therapeutischen Ziele ergeben sich für den Therapeuten somit konkrete Handlungsanweisungen zur Beziehungsgestaltung. Dieser Entwicklung liegt die Annahme zugrunde, dass die therapeutische Beziehung nicht ausschließlich als Basis für den Einsatz spezifischer Techniken fungiert, sondern durch die Arbeit mit der Therapeut-Patient-Beziehung per se Veränderungen bewirkt werden können. Besonders die DBT, die Schematherapie und das CBASP betonen - im Gegensatz zur ersten und zweiten Welle der VT - die therapeutische Beziehung als wesentlichen Aspekt zur Überwindung störungsimmanenter interpersoneller Schwierigkeiten. Entwickelt wurden diese Ansätze vor allem für persistierende psychische Störungen, die mit interaktionellen Schwierigkeiten und Problemen einhergehen [Linehan, 1993; McCullough, 2003; Young et al., 2003]. Daraus lässt sich schlussfolgern, dass die Anforderungen an die Beziehungsgestaltung mit zunehmendem Schweregrad interaktioneller Probleme [z. B. Persönlichkeitsstörungen; Forster et al., 2014] komplexer werden und spezifischere Konzepte hilfreich erscheinen. Entsprechend dem von Zilcha-Mano [2017] vorgeschlagenen Modell, bilden sich die schwerwiegenden interaktionellen Schwierigkeiten eines Patienten zunächst in der trait-Komponente der therapeutischen Allianz ab. Diese Patienten haben gravierende Probleme, zufriedenstellende und gefestigte Beziehungen zu anderen aufzubauen und aufrechtzuerhalten, was sich auch in der Beziehung zum Therapeuten niederschlägt und sich durch geringe Werte in der therapeutischen Allianz abbilden lässt [Constantino et al., 2010; Grosse Holtforth et al., 2014; Renner et al., 2012]. Für diese Patienten sind möglicherweise die Veränderungen in der therapeutischen Allianz (stateKomponente) entscheidend, um positive Veränderungen in Bezug auf problematische Interaktionsmuster zu erreichen, was sich wiederum in den interpersonellen Beziehungen außerhalb des Therapiekontextes widerspiegelt [Zilcha-Mano und Errázuriz, 2015; Zilcha-Mano et al., 2018b]. Die vorgeschlagene Unterscheidung erscheint wichtig für eine empirisch begründete differentielle Beziehungsgestaltung. Dabei können die trait-Komponenten Hinweise liefern, wie der Therapeut seine Beziehungsgestaltung mit bestimmten Patienten für den Aufbau der therapeutischen Allianz gestaltet. Beispielsweise kann bei einem sozial phobischen Patienten eine sehr ressourcenorientierte und empathische Haltung des Therapeuten in der Eingangsphase dazu führen, dass im Kontext der therapeutischen Beziehung selbst ein Bewer- tungsdruck erzeugt wird. Dies kann als angstauslösend erlebt werden und in der Konsequenz zur Vermeidung des therapeutischen Beziehungsangebots führen. Demgegenüber kann das beschriebene Beziehungsangebot für einen depressiven Patienten in der Eingangsphase sehr entlastend und förderlich sein.

In der KVT wurde traditionell vor allem die traitKomponente der Allianz hervorgehoben [Beck et al., 1979; Castonguay et al., 2010]. Allerdings haben Handlungsempfehlungen mit Betonung der state-Komponente seit einigen Jahren auch in der KVT keinen Seltenheitswert mehr: Die therapeutische Beziehung kann somit ein machtvolles Werkzeug für die Veränderung bzw. Neubewertung von dysfunktionalen Grundannahmen in Bezug auf die eigene Person und Andere sein. Die Beziehungsgestaltung orientiert sich dabei an einer fortlaufenden Fallkonzeption, wobei die Bedürfnisse und Voraussetzungen des Patienten von entscheidender Bedeutung sind. Besonders bei interaktionellen Schwierigkeiten kann der Einbezug früherer Beziehungserfahrungen des Patienten hilfreich sein, wobei die Adaptation der therapeutischen Beziehungsgestaltung im therapeutischen Prozess generell das Potential für unabhängige Therapieeffekte birgt [Kazantzis et al., 2018].

Die Indikation für die Gestaltung der therapeutischen Beziehung sollte dementsprechend auf Basis einer fortlaufenden - sich aus im Zuge der therapeutischen Arbeit gewonnenen Informationen speisenden - Fallkonzeption gestellt werden und sich auf empirische Befunde stützen [Cronin et al., 2015]. Belege für den Nutzen einer individualisierten Fallkonzeption in Bezug auf die therapeutische Allianz lassen sich vor allem in Dismantlingstudien zeigen. In einer Studie zum plananalytischen Vorgehen im Kontext der motivorientierten Beziehungsgestaltung mit insgesamt 85 eingeschlossenen BPS-Patienten fanden Kramer et al. [2014], dass eine generelle psychiatrische Behandlung mit einem motivorientierten Vorgehen im Gegensatz zur alleinigen generellen psychiatrischen Behandlung die Entwicklung der therapeutischen Allianz begünstigte. Zusätzlich zeigten sich Effekte auf die Reduktion der allgemeinen Symptomatik sowie interpersoneller und sozialer Probleme. Allerdings fanden sich keine Unterschiede in Bezug auf die störungsspezifische Symptomatik, was unter anderem mit dem relativ kurzen Behandlungszeitraum (10 Sitzungen) begründet werden kann. Jedoch verdeutlichen die Ergebnisse den Nutzen einer fundierten, individualisierten und theoriegeleiteten Fallkonzeption im Hinblick auf den Aufbau einer tragfähigen therapeutischen Allianz.

Besonders deutlich wird dies, wenn man sich die Motivation auf Patientenseite vergegenwärtigt, neue Verhaltensmuster zu erlernen. Solche Veränderungen fallen angesichts eingefahrener, automatisierter Schemata und Verhaltensweisen naturgemäß schwer - auch wenn diese 
Schemata und Verhaltensweisen mittlerweile langfristig dysfunktional geworden sind und nicht mehr den eigentlich legitimen Bedürfnissen des Patienten dienen. Interessante Ergebnisse hierzu finden sich in einer Studie zu Äußerungen von Patienten mit einer generalisierten Angststörung bezüglich der intrinsischen Motivation zur Veränderung in den ersten Therapiesitzungen [Sijercic et al., 2016]. Die Autoren berichten, dass Patienten, die ihre Argumente und Gegenargumente auf den interpersonellen Kontext der Therapie - also auch auf den Therapeuten - bezogen, entsprechend schlechtere Therapieergebnisse aufwiesen als Patienten, die ihre ausgedrückte Ambivalenz hinsichtlich möglicher Veränderungen nicht in einen interpersonellen Kontext setzten. Diese Studie verdeutlicht, dass es, insbesondere bei Patienten mit hohem Reaktanzniveau, oft eines "Beziehungskredits" [vgl. den entsprechenden Ansatz von Rainer Sachse, 2006] bedarf, der initial aufgebaut wird und dann zu einem späteren Zeitpunkt ("wenn es wirklich unangenehm wird") zur konfrontativen Veränderungsarbeit eingesetzt werden kann.

Ausgehend von den Aspekten der Indikation und Individualisierung sollte die differentielle Gestaltung der Beziehung vor allem dann spezifisch erfolgen, wenn interaktionelle Schwierigkeiten und Muster im Rahmen der Beziehungsarbeit adressiert werden. Ein Grundprinzip der Psychotherapie - nämlich die Vermittlung korrigierender Erfahrungen [Alexander und French, 1946] - kann dadurch umgesetzt werden, dass diese durch heilsame Beziehungserfahrungen anhand der therapeutischen Beziehung hergestellt werden. Im Folgeschritt kann dann bisheriges Problemverhalten "im richtigen Leben" modifiziert oder aufgegeben werden. Hier sind aus verhaltenstherapeutischer Perspektive interpersonelle Lerntherapien gefragt, bei denen der Therapeut unter Umständen auch gezielt seine eigenen zwischenmenschlichen Gefühlsregungen dem Patienten rückmeldet (wie beim DPI in CBASP), oder bei denen er als zwischenmenschliches Modell fungiert und in der Therapie sehr aktiv für einen verlässlichen zwischenmenschlichen Rahmen sorgt (z. B. in der Schematherapie: Der Therapeut als Beispiel eines "gesunden Erwachsenenmodus").

Der Aufbau und die Arbeit mit einer tragfähigen therapeutischen Allianz sind somit von hoher Bedeutung für die drei vorgestellten Ansätze zur Behandlung von Patienten mit frühen traumatisierenden Beziehungserfahrungen, was durch erste Studien untermauert wird [DBT: Bedics et al., 2015; Stiglmayr et al., 2014; Schematherapie: Spinhoven et al., 2007; CBASP: Arnow et al., 2013; Constantino et al., 2016; Santiago et al., 2005]. Die authentische Selbstöffnung des Therapeuten scheint dabei als ein wesentliches Element der Beziehungsgestaltung zu fungieren, wobei sich Form und Dosis der Selbstöffnung in der Konzeption der Ansätze unterscheidet [Köhler et al., 2017]. Dabei umfasst die Selbstöffnung in der Schematherapie vor allem das Offenlegen positiver Emotionen der Akzeptanz und Wärme, um frustrierte Grundbedürfnisse der Patienten zu überwinden. In der DBT ist Selbstöffnung im Rahmen von Akzeptanz- und Veränderungsstrategien vor allem indiziert, um mit autoaggressivem und destruktivem Patientenverhalten umzugehen, welches den therapeutischen Prozess behindert. Dabei umfasst die Selbstöffnung sowohl negative als auch positive Emotionen. Im CBASP dient die Offenlegung positiver wie auch negativer Gefühle vor allem dem transparenten Erleben von zwischenmenschlichen Konsequenzen. Der Therapeut verhält sich während der Sitzungen oft entgegen der auf früheren Beziehungserfahrungen beruhenden Erwartungen des Patienten, was heilsame Beziehungserfahrungen ermöglichen kann.

Die Betonung der persönlichen - und damit "echten" - Rückmeldung eigener Gefühle durch den Therapeuten ist dabei nicht unerheblich, wie eine ausführliche Gegenüberstellung zweier DBT-Behandlungen in einer zusammenfassenden Falldarstellung eindrucksvoll verdeutlicht [Burckell und McMain, 2011]. Beide Patienten mit einer BPS zeigten initial niedrige Werte in der therapeutischen Allianz (Working Alliance Inventory) und feindseliges Verhalten. Während sich jedoch die Werte in der Allianz im Verlauf der Therapie für einen Patienten verbesserten, blieben die Werte im anderen Fall konstant auf dem initial niedrigen Niveau. Diese Verläufe spiegelten sich auch in den Ergebnissen der Therapie wider. Im Fall der konstant niedrigen Allianzwerte fühlte sich die Therapeutin stark vom Patienten angegriffen und verletzt, weshalb es ihr nur sehr schwer möglich war, Mitgefühl für den Patienten aufzubringen, was wiederum die "echte" bzw. authentische Validierung des Patienten durch die Therapeutin beeinflusste. Validierungsversuche wurden vom Patienten wiederholt durch feindselige Angriffe abgeblockt. Infolgedessen verstärkten sich bei der Therapeutin Gefühle von Demoralisierung und Hoffnungslosigkeit in Bezug auf den Patienten und die gemeinsame therapeutische Arbeit. Die Autoren unterstreichen an dieser Stelle, dass Therapeutin und Patient über den gesamten Therapieverlauf hinweg in einer Sackgasse steckten und nicht in der Lage waren, sich aus den polarisierten Positionen heraus zu bewegen. Dementsprechend gab es keine "echte" emotionale Verbindung zwischen der Therapeutin und dem Patienten.

Die skizzierte Falldarstellung illustriert anschaulich eine Reihe von Fragen, die es im Zusammenhang mit einer empirisch begründeten differentiellen und individualisierten Beziehungsgestaltung zu klären gilt: 
(1) Welche Elemente der therapeutischen Beziehung und Beziehungsgestaltung sind in welcher

Kombination für welchen Patienten hilfreich?

In der zusammengefassten Falldarstellung wird deutlich, dass Aspekte der Selbstöffnung eng mit einer authentischen Beziehung zwischen Patient und Therapeut verknüpft sind. Dieser Aspekt einer authentischen, "echten", emotionalen Bindung lässt sich am ehesten durch die BOND-Komponente der Working Alliance [Bordin, 1979] abbilden. Diese überschneidet sich wiederum stark mit dem Konstrukt der "real relationship" - der existierenden persönlichen Beziehung zwischen zwei Menschen, die den Grad an präsenter Aufrichtigkeit sowie einer realistischeren Wahrnehmung voneinander widerspiegelt [Gelso et al., 2018]. Möglicherweise sind diese Elemente der therapeutischen Beziehung entscheidender für den Therapieerfolg bei der Behandlung von Patienten mit Kindesmisshandlungserfahrungen. Zukünftige Studien könnten somit beispielsweise Aufschluss über die Wirkungsweise der therapeutischen Beziehung liefern, indem sie diese Aspekte besonders für Patienten mit persistierenden psychischen Störungen und Kindesmisshandlung stärker berücksichtigen. Dabei gilt es auch, mögliche negative Effekte nicht aus dem Blickfeld zu verlieren. In Bezug auf die Beziehungsgestaltung können negative Effekte im Sinne einer Abhängigkeit des Patienten von Behandlung und Therapeut auftreten. Eine damit verbundene passive Haltung des Patienten kann sich dementsprechend negativ auf Behandlungsdauer und Rückfallraten auswirken [Brakemeier et al., 2018; Geurtzen et al., 2019]. Bisher werden negative Zusammenhänge zwischen einer misslungenen therapeutischen Beziehung und dem Behandlungserfolg vor allem in psychodynamischen Kreisen diskutiert [Knox, 2019].

Um der Beantwortung der formulierten Frage näher zu kommen, können möglicherweise auch experimentelle Forschungsdesigns zur therapeutischen Beziehungsgestaltung hilfreich sein. In einem entsprechenden Pilotprojekt wurden im Kontext eines einmaligen Beratungsgesprächs für Studierende zum Thema interpersonelle Konflikte zwei zuvor definierte Beziehungsgestaltungsstile (sachorientiert vs. beziehungsorientiert) in einem randomisierten Zweigruppendesign miteinander verglichen [Schamong et al., 2019]. Der Fokus dieser explorativen Studie lag dabei vor allem auf der Durchführbarkeit einer solchen experimentellen Studie, wobei erste Ergebnisse auf eine gute bis sehr gute Adhärenz der jeweiligen Berater zu den zwei Bedingungen durch objektive Rater hinweisen.

(2) Welche Bedeutung hat die therapeutische Allianz zu welchem Zeitpunkt für welchen Patienten?

Die beiden oben erwähnten Fallbeispiele verdeutlichen eindrücklich den Wert des von Zilcha-Mano [2017] vorgeschlagenen Zwei-Komponenten-Modells, in dem die Veränderungen in der therapeutischen Allianz (stateKomponente) bei einigen Patienten mitentscheidend für den Therapieerfolg sein können und bei anderen nicht. Dabei bedeutet eine initial schlechtere therapeutische Allianz (trait-Komponente) nicht notwendigerweise auch ein schlechtes Therapieergebnis. Auf dem Weg zu einer evidenzbasierten differentiellen Beziehungsgestaltung erscheint es daher wichtig, Moderatoren für den Zusammenhang zwischen den beiden Komponenten und dem Therapieerfolg $\mathrm{zu}$ identifizieren. Zilcha-Mano et al. [2018c] konnten in einer Sekundäranalyse von 185 Patienten, die eine ambulante KVT-Behandlung erhalten hatten, mithilfe von "machine-learning"-Algorithmen signifikante Interaktionen der beiden Komponenten mit spezifischen interpersonellen Charakteristika der Patienten zeigen. Bei Patienten, die sich selbst als kalt bzw. zu abweisend/distanziert beschrieben und in ähnlicher Weise auch von ihren Therapeuten eingeschätzt wurden, hatte die Verbesserung der therapeutischen Allianz im Behandlungsverlauf einen deskriptiv größeren Effekt auf den Therapieerfolg als bei Patienten, die sich als zu kalt bzw. $\mathrm{Zu}$ abweisend/distanziert beschrieben haben. $\mathrm{Zu}-$ gleich zeigte sich für die trait-Komponente der Allianz ein größerer Effekt auf den Therapieerfolg, wenn die Patienten sich selbst als nicht zu abweisend/kalt sowie übermäßig aufdringlich beschrieben. Die Ergebnisse liefern somit einen Beitrag zur Beantwortung der Frage, ob es für den individuellen Patienten hilfreich ist, die therapeutische Allianz im Behandlungsverlauf stärker zu fokussieren und aktiv mit dieser zu arbeiten.

Darauf aufbauend erscheint es wichtig, die Frage zu klären, wann die aktive therapeutische Beziehungsgestaltung im Hinblick auf die individuell ausformulierten Therapieziele am effektivsten ist. Hierzu fand eine Studie von Zilcha-Mano et al. [2018a] einen moderierenden Einfluss der Lebenszufriedenheit auf den Zusammenhang zwischen positiven Veränderungen in der Allianz für eine bestimmte Therapiesitzung und dem Behandlungserfolg in der darauffolgenden Sitzung. Dies würde bedeuten, dass die aktive Fokussierung auf die Verbesserung der Allianz einen größeren Effekt auf den Therapieerfolg haben kann, wenn die Patienten zum entsprechenden Zeitpunkt zumindest nicht ganz unzufrieden mit ihrer aktuellen Lebenssituation sind.

\section{(3) Was genau zeichnet effektive Therapeuten in} Bezug auf die Formung und Aufrechterhaltung der therapeutischen Allianz aus?

Für eine empirisch begründete differentielle Beziehungsgestaltung kann der Einbezug der Therapeutenperspektive zunächst einmal auf Diskrepanzen oder Konsens in der Wahrnehmung der therapeutischen Allianz hinweisen. Studien konnten zeigen, dass sich die Einschät- 
zungen der therapeutischen Allianz von Therapeut und Patient einander mit zunehmendem Behandlungsverlauf anglichen [Coyne et al., 2018; Laws et al., 2017]. Basierend auf zwei Fremdeinschätzungsratings zeigten sich jedoch inkonsistente Befunde für die Annahme, dass stärker konvergierende Allianzverläufe auch mit einer stärkeren Symptomreduktion verbunden waren [Constantino et al., 2017]. Hierbei gilt es zu berücksichtigen, dass Therapeuten und Patienten wahrscheinlich unterschiedliche Kriterien für die Beurteilung der therapeutischen Allianz heranziehen [Kivlighan et al., 2014]. Diskrepanzen in der Wahrnehmung der therapeutischen Allianz bedeuten dementsprechend nicht automatisch Uneinigkeit im negativen Sinne, sondern sind vorrangig als Hinweise $\mathrm{zu}$ verstehen, dass die Thematisierung der therapeutischen Beziehung möglicherweise nützlich und hilfreich sein könnte [Flückiger et al., 2018].

Darüber hinaus veranschaulichen die zwei dargestellten Fälle praxisnah die Befunde von Schiefele et al. [2017] zu Therapeuteneffekten: Je komplexer die Symptomatik eines Patienten ist, desto wichtiger wird die Person des Therapeuten [Johns et al., 2019]. Effektive Therapeuten scheinen sich vor allem durch professionell verfeinerte interpersonelle Fähigkeiten auszuzeichnen [u. a. Anderson et al., 2009; Schöttke et al., 2017], die möglicherweise in ihrem persönlichen Leben und ihrer Bindungsgeschichte ihren Ursprung haben [Heinonen und NissenLie, 2020]. Wie die skizzierte Falldarstellung von Burckell und McMain [2011] veranschaulicht, sind ist das Erkennen und die Reflektion einer für den therapeutischen Prozess wenig förderlichen therapeutischen Beziehung allein nicht immer ausreichend, um für den Einzelfall passende therapeutische Reaktionen abzuleiten. Die Reflektion sollte deshalb die persönlichen Grundannahmen und das Wertesystem des jeweiligen Therapeuten miteinschließen [Kazantzis et al., 2018]. Dadurch kann die Wahrscheinlichkeit erhöht werden, dass der jeweilige Therapeut die abgeleiteten Handlungsoptionen in der Durchführung für sich auch als passend empfindet, wodurch er wiederum authentisch bleiben kann.

Insgesamt finden der dyadische Charakter der therapeutischen Beziehung genauso wie die Person des Therapeuten jedoch noch zu wenig Berücksichtigung in der gegenwärtigen Forschung. Neben der konsequenten Berücksichtigung der Therapeutenvariable in geschachtelten Designs könnten sich zukünftige Studien u. a. verstärkt mit aufgezeichnetem Videomaterial einzelner Sitzungen oder sogar ganzer Behandlungen beschäftigen, um vergleichbare kritische Situationen [Anderson et al., 2009] und die unmittelbaren Reaktionen der einzelnen Therapeuten darauf näher zu beleuchten und zu untersuchen.

\section{(4) Wie kann eine evidenzbasierte differentielle}

Beziehungsgestaltung in der Praxis umgesetzt werden?

Die Implikationen von Forschungsbefunden zur therapeutischen Beziehung und ihrer Gestaltung für die praktische Arbeit sind oft schnell formuliert. Für deren Implementation braucht es jedoch anwendungsfreundliche Konzepte, die für den Praktiker einen erkennbaren Mehrwert in der individuellen Arbeit mit einem bestimmten Patienten liefern. Das aus der Forschung zu Allianzbrüchen und ihrer Reparatur [engl. ruptures and repairs; Safran und Muran, 2000] entwickelte allianzfokussierte Training [alliance-focused training; Eubanks-Carter et al., 2015] zielt darauf ab, die Fähigkeiten von Therapeuten im Erkennen, Tolerieren und Aushandeln von Allianzbrüchen zu verbessern. Dieses Training kann als konzeptionelle Umsetzung einer evidenzbasierten differentiellen Beziehungsgestaltung verortet werden. Im Gegensatz zum Ergebnis einer früheren Metaanalyse [Safran et al., 2011] zeigte sich in einer zweiten Metaanalyse mit sechs eingeschlossen Studien jedoch kein signifikant positiver Zusammenhang zwischen einem zusätzlichen allianzfokussierten Training und dem Therapieerfolg [Eubanks et al., 2018]. Moderatoranalysen ergaben, dass der Zusammenhang eines allianzfokussierten Trainings mit dem Therapieerfolg größer war, wenn die Stichprobe weniger Patienten mit Persönlichkeitsstörungen beinhaltete, das Training enger auf eine KVT abgestimmt war und die Behandlung kürzer war. Demnach ist allein der Fokus auf bestimmte Elemente der therapeutischen Beziehung in Training und Supervision oft nicht ausreichend. Supervision und Training sollten demnach - auf den Einzelfall abgestimmt - das Zusammenspiel verschiedener Elemente der Beziehungsgestaltung im Kontext unterschiedlicher Situationen im Blick haben und mit evidenzbasierten Handlungsempfehlungen abgleichen. Konsequenterweise sollte hierbei die kontinuierliche Erfassung der therapeutischen Allianz aus Patientenperspektive als Basis für die Supervision dienen [Reese et al., 2009]. Die Feedbackforschung liefert hierzu grundlegende Ideen, wie die Realisierung einer evidenzbasierten differentiellen Beziehungsgestaltung aussehen könnte [Lutz et al., 2015; Schiepek et al., 2018].

Neben Feedbacksystemen steigt die Zahl vielversprechender Vorhersagemodelle zur Optimierung psychotherapeutischer Behandlungen mit dem Ziel der Maximierung des individuellen Therapieerfolges [Rubel und Lutz, 2016]. Die Integration von Variablen zur therapeutischen Beziehung einschließlich ihrer verschiedenen Elemente kann dabei möglicherweise die Vorhersagekraft dieser Modelle verbessern. Andererseits könnten Modelle, die auf eine Handlungsempfehlung für den Einsatz spezifischer Techniken oder Strategien abzielen [Cohen und DeRubeis, 2018; Rubel et al., 2018], auch für Elemente der Beziehungsgestaltung nützlich sein. In solchen Modellen 
sollte allerdings auch die Person des Therapeuten berücksichtigt werden. Die Passung von Elementen der Beziehungsgestaltung zur Person des Therapeuten und seinen spezifischen Charakteristika sollte nicht unterschätzt werden. Dabei kennen wir bei Weitem noch nicht alle prädikativen Variablen und deren Zusammenspiel untereinander im Hinblick auf die Therapeut-Patient-Dyade.

Noch stecken solche Modelle und Algorithmen in den Kinderschuhen. Ihr Potenzial in Bezug auf die Maximierung des Therapieerfolgs unter Einbezug einer evidenzbasierten differentiellen Gestaltung der Beziehung ist jedoch deutlich erkennbar.

\section{Fazit und Ausblick}

Alle dargestellten Ansätze bauen auf eine konzeptionell ausformulierte therapeutische Beziehungsgestaltung, wobei unterschiedliche Schwerpunkte betont werden. Die hier beschriebenen Ausführungen und Ansätze sind dabei keinesfalls als vollständig zu verstehen, wobei besonders die Beziehungsgestaltung und ihre Konzeptualisierung in unterschiedlichen Settings (z. B. Gruppentherapie, Paartherapie), die Bedeutung der Beziehung aus der Perspektive anderer (psychologischer) Disziplinen, die Frage nach der statistischen Modellierung oder die Betrachtung von Beziehungserwartungen in Verbindung mit der Placebo-Forschung wichtige Aspekte darstellen, die über die vorliegende Übersichtsarbeit hinausgehen. Um korrigierende interpersonelle Erfahrungen zu fördern und zu realisieren, formuliert zum Beispiel auch die emotionsfokussierte Therapie [Greenberg, 2015] ein strukturiertes Vorgehen. Explizit werden dabei die Emotionen des Patienten in der therapeutischen Arbeit und der Beziehungsgestaltung adressiert, wobei der Therapeut die Rolle eines "Emotionscoachs" übernimmt. Andere Ansätze stellen die Individualität der therapeutischen Beziehung und ihrer Gestaltung besonders in den Vordergrund, indem beispielsweise der Therapeut in der Akzeptanz- und Committmenttherapie ausdrücklich dazu aufgefordert wird, individualisierte Interventionen $\mathrm{zu}$ erarbeiten und anzuwenden [Hayes und Lillis, 2012].

Historisch gewachsen ist in der VT der Aspekt der individuellen Gestaltung der Beziehung, welche dem Therapeuten intuitive Freiheit in der Realisierung gewährt. Dies mag damit zu tun haben, dass der therapeutischen Beziehung zunächst wenig Beachtung geschenkt wurde, was sicherlich auch dem damaligen Zeitgeist mit dem Bedürfnis nach einer klaren Abgrenzung zur psychodynamischen Schulen geschuldet ist. Dieses Vakuum hat es jedoch möglich gemacht, dass wir in der Realisierung der therapeutischen Beziehungsgestaltung in der VT heute von einer Vielzahl an theoretisch fundierten Konzepten profitieren können. Diese Konzepte im Sinne einer evi- denzbasierten differentiellen Beziehungsgestaltung individuell, indiziert, und spezifisch nutzbar zu machen, ist deshalb Anliegen und Aufgabe einer individualisierten Psychotherapie [vgl. Brakemeier und Herpertz, 2019]. In diesem Zusammenhang können vor allem integrative Modelle die therapeutische Sicht auf den individuellen Patienten bereichern [Rief und Strauß, 2018].

Allerdings wäre es ein fataler Trugschluss zu denken, man könne sich in der therapeutischen Arbeit allein auf die Beziehung verlassen. Ein vielfältiges therapeutisches Repertoire an aufeinander aufbauenden Techniken ist essentiell für eine erfolgreiche psychotherapeutische Behandlung.

Dementsprechend bedeutet Indikation keine Entweder-oder-Entscheidung, sondern betont stattdessen die Notwendigkeit einer kontinuierlichen Überprüfung des Therapiefortschritts auf der Basis einer fortlaufenden individuellen Fallkonzeption. Dies beinhaltet, dass zum angemessenen Einsatz einer evidenzbasierten differentiellen Beziehungsgestaltung auch bedacht werden muss, wann welche Beziehungsgestaltungselemente und -kompetenzen nicht zum Einsatz kommen sollten. Nicht immer scheint eine aktive Beziehungsgestaltung, die spezifischen Therapiezielen dient, notwendig oder sogar hilfreich für den Therapieerfolg.

Im Angesicht der wachsenden Zahl von Online-Therapieangeboten sind diese Überlegungen von besonderer Bedeutung. Dabei wird sich zeigen, ob eine therapeutische Beziehungsgestaltung, die spezifischen Zielen dient (z. B. einer korrigierenden Beziehungserfahrung), auch online realisiert werden kann.

\section{Statement of Ethics}

Für diese Übersichtsarbeit war keine Ethik-Erklärung nötig.

\section{Conflict of Interest Statement}

Die Autoren erklären, dass kein Interessenskonflikt besteht.

\section{Funding Sources}

Es bestand keine finanzielle Unterstützung.

\section{Author Contributions}

S.B. und S.K. erarbeiteten die erste Version des Manuskriptes, welche von S.B. finalisiert wurde. Die konzeptionelle Erweiterung und Überarbeitung des Manuskriptes erfolgte durch S.B. und E.-L.B. unter Mitwirkung aller Autoren, welche substantiell kritische Anmerkungen und Rückmeldungen im Zuge der Finalisierung des Artikels gaben. 


\section{Literatur}

Alexander F, French TM. Psychoanalytic therapy. New York: Ronald Press; 1946.

Anderson T, Ogles BM, Patterson CL, Lambert MJ, Vermeersch DA. Therapist effects: facilitative interpersonal skills as a predictor of therapist success. J Clin Psychol. 2009 Jul; 65(7):755-68

Arnow BA, Steidtmann D, Blasey C, Manber R, Constantino MJ, Klein DN, et al. The relationship between the therapeutic alliance and treatment outcome in two distinct psychotherapies for chronic depression. J Consult Clin Psychol. 2013 Aug;81(4):627-38.

Bandura A. Principles of behavior modification. New York: Holt, Rinehart and Winston; 1969.

Barkham M, Lutz W, Lambert MJ, Saxon D. Therapist effects, effective therapists, and the law of variability [Internet] Castonguay LG, Hill $\mathrm{CE}$, editors. How and why are some therapists better than others?: Understanding therapist effects. Washington: American Psychological Association; 2017. pp. 13-36.

Barnicot K, Katsakou C, Bhatti N, Savill M, Fearns N, Priebe S. Factors predicting the outcome of psychotherapy for borderline personality disorder: a systematic review. Clin Psychol Rev. 2012 Jul;32(5):400-12.

Beck AT, Rush AJ, Shaw BE, Emery G. Cognitive therapy of depression. New York: Guilford Press; 1979.

Bedics JD, Atkins DC, Harned MS, Linehan MM The therapeutic alliance as a predictor of outcome in dialectical behavior therapy versus nonbehavioral psychotherapy by experts for borderline personality disorder. Psychotherapy (Chic). 2015 Mar;52(1):67-77.

Bordin ES. The generalizability of the psychoanalytic concept of the working alliance. Psychotherapy (Chic). 1979;16(3):252-60.

Brakemeier EL. Schwierige Situationen in der modernen Psychotherapie. CBASP, DBT, MBT und Schematherapie. Beltz VideoLearning. Weinheim: Beltz; 2019.

Brakemeier EL, Fassbinder E, Stiglmayr C. Spezielle Beziehungsgestaltungstechniken aus neueren verhaltenstherapeutischen Methoden. In: Brakemeier EL, Jacobi F, editors. Verhaltenstherapie in der Praxis. Weinheim: Beltz; 2017. pp. 76-92.

Brakemeier EL, Frase L. Interpersonal psychotherapy (IPT) in major depressive disorder. Eur Arch Psychiatry Clin Neurosci. 2012 Nov; 262(S2 Suppl 2):S117-21.

Brakemeier EL, Herpertz SC. Innovative Psychotherapieforschung: Auf dem Weg zu einer evidenz- und prozessbasierten individualisierten und modularen Psychotherapie. Nervenarzt. 2019 Nov;90(11):1125-34.

Brakemeier EL, Herzog P, Radtke M, Schneibel R, Breger V, Becker M, et al. CBASP als stationäres Behandlungskonzept der therapieresistenten chronischen Depression: Eine Pilotstudie zum Zusammenhang von Nebenwirkungen und Therapieerfolg. Psychother Psychosom Med Psychol. 2018;68:399-407.

Brakemeier EL, Guhn A, Normann C. Praxisbuch CBASP. Behandlung chronischer Depression und Modifikationen der traditionellen CBASP-Therapie. Weinheim: Beltz; 2021.
Burckell LA, McMain S. Contrasting clients in dialectical behavior therapy for borderline personality disorder: "Marie" and "Dean," two cases with different alliance trajectories and outcomes. Pragmat Case Stud Psychother. 2011; 7(2): 246-67. https://doi. org/10.14713/pcsp.v7i2.1090.

Caspar F. Beziehungen und Probleme verstehen: eine Einführung in die psychotherapeutische Plananalyse. Göttingen: Hogrefe; 2018. https://doi.org/10.1024/85625-000.

Caspar F, Belz M. Motivorientierte Beziehungsgestaltung. In: Brakemeier EL, Jacobi F, editors. Verhaltenstherapie in der Praxis. Weinheim: Beltz; 2017. p. 54-65.

Castonguay LG, Constantino MJ, McAleavey AA, Goldfried MR. The therapeutic alliance in cognitive-behavioral therapy. In: Muran JC, Barber JP, editors. The therapeutic alliance: an evidence-based guide to practice. New York: Guilford Press; 2010. p. 263-85.

Cohen R. Verhaltenstherapie zu Beginn der achtziger Jahre. Psychol Rundsch. 1984;35: $1-9$.

Cohen ZD, DeRubeis RJ. Treatment Selection in Depression. Annu Rev Clin Psychol. 2018 May;14(1):209-36.

Constantino MJ, Coyne AE, Luukko EK, Newkirk K, Bernecker SL, Ravitz P, et al. Therapeutic alliance, subsequent change, and moderators of the alliance-outcome association in interpersonal psychotherapy for depression. Psychotherapy (Chic). 2017 Jun;54(2):125-35.

Constantino MJ, Laws HB, Coyne AE, Greenberg RP, Klein DN, Manber R, et al. Change in patients' interpersonal impacts as a mediator of the alliance-outcome association in treatment for chronic depression. J Consult Clin Psychol. 2016 Dec;84(12):1135-44.

Constantino MJ, Schwaiger EM, Smith JZ, DeGeorge J, McBride C, Ravitz P, et al. Patient interpersonal impacts and the early therapeutic alliance in interpersonal therapy for depression. Psychotherapy (Chic). 2010 Sep; 47(3):418-24.

Coyne AE, Constantino MJ, Laws HB, Westra HA, Antony MM. Patient-therapist convergence in alliance ratings as a predictor of outcome in psychotherapy for generalized anxiety disorder. Psychother Res. 2018 Nov;28(6): 969-84.

Cronin TJ, Lawrence KA, Taylor K, Norton PJ, Kazantzis N. Integrating between-session interventions (homework) in therapy: the importance of the therapeutic relationship and cognitive case conceptualization. J Clin Psychol. 2015 May;71(5):439-50.

Del Re AC, Flückiger C, Horvath AO, Symonds $\mathrm{D}$, Wampold BE. Therapist effects in the therapeutic alliance-outcome relationship: a restricted-maximum likelihood meta-analysis. Clin Psychol Rev. 2012 Nov;32(7):642-9.

Ellis A, Hoellen B. Die rational-emotive Verhaltenstherapie: Reflexionen und Neubestimmungen. München: Pfeiffer; 1997.

Eubanks CF, Muran JC, Safran JD. Alliance rupture repair: A meta-analysis. Psychotherapy (Chic). 2018 Dec;55(4):508-19.
Eubanks-Carter C, Muran JC, Safran JD. Alliance-focused training. Psychotherapy (Chic). 2015 Jun;52(2):169-73.

Falkenström F, Granström F, Holmqvist R. Therapeutic alliance predicts symptomatic improvement session by session. J Couns Psychol. 2013 Jul;60(3):317-28.

Fiedler P. Varianten psychotherapeutischer Beziehung: Transdiagnostische Befunde, Konzepte, Perspektiven. Lengerich: Pabst Science Publishers; 2018.

Flückiger C. Bedeutung der Arbeitsallianz für die Psychotherapie. Z Klin Psychol Psychother. 2018;47:119-25.

Flückiger C, Del Re AC, Horvath AO, Symonds D, Ackert M, Wampold BE. Substance use disorders and racial/ethnic minorities matter: a meta-analytic examination of the relation between alliance and outcome. J Couns Psychol. 2013 Oct;60(4):610-6.

Flückiger C, Del Re AC, Wampold BE, Horvath AO. The alliance in adult psychotherapy: A meta-analytic synthesis. Psychotherapy (Chic). 2018 Dec;55(4):316-40.

Flückiger C, Del Re AC, Wampold BE, Symonds $\mathrm{D}$, Horvath AO. How central is the alliance in psychotherapy? A multilevel longitudinal meta-analysis. J Couns Psychol. 2012a Jan; 59(1):10-7.

Flückiger C, Del Re AC, Wampold BE, Znoj H, Caspar F, Jörg U. Valuing clients' perspective and the effects on the therapeutic alliance: a randomized controlled study of an adjunctive instruction. J Couns Psychol. 2012b Jan; 59(1):18-26.

Flückiger C, Holtforth MG. Ressourcenaktivierung und motivorientierte Beziehungsgestaltung: Bedürfnisbefriedigung in der Psychotherapie[Internet] Frank R, editor. Therapieziel Wohlbefinden. Berlin: Springer; 2007. p. 33-42.

Forster C, Berthollier N, Rawlinson D. A systematic review of potential mechanisms of change in psychotherapeutic interventions for personality disorder. J Psychol Psychother. 2014; 4(1). DOI: 10.4172/2161-0487.1000133.

Frank JD, Frank JB. Persuasion and healing: comparative study of psychotherapy. Baltimore: Johns Hopkins University Press; 1993.

Fuertes JN, Anand P, Haggerty G, Kestenbaum M, Rosenblum GC. The physician-patient working alliance and patient psychological attachment, adherence, outcome expectations, and satisfaction in a sample of rheumatology patients. Behav Med. 2015;41(2):60-8.

Gelso CJ, Kivlighan DM, Markin RD. The real relationship and its role in psychotherapy outcome: A meta-analysis. Psychotherapy (Chic). 2018 Dec;55(4):434-44.

Geurtzen N, Keijsers GP, Karremans JC, Hutschemaekers GJ. Care dependency may help and hurt psychological treatment: A treatmentanalogue study with students in clinical training. J Psychother Integration. 2019;29(4): 374-88.

Goldfried MR, Davison GC. The therapeutic relationship. In: Goldfried MR, Davison GC, editors. Clinical Behavior therapy. New York: Holt, Rinehart \& Winston; 1976. 
Grawe K. Psychologische Therapie. Göttingen: Hogrefe; 2000.

Greenberg LS. Emotion-focused therapy: Coaching clients to work through their feelings. 2nd ed. Washington (DC): American Psychological Association; 2015. https://doi. org/10.1037/14692-000.

Greenson RR. The working alliance and the transference neurosis. PsycNET Psychoanal Q. 1965 Apr;34(2):155-81.

Grosse Holtforth M, Altenstein D, Krieger T, Flückiger C, Wright AG, Caspar F. Interpersonal differentiation within depression diagnosis: relating interpersonal subgroups to symptom load and the quality of the early therapeutic alliance. Psychother Res. 2014; 24(4):429-41.

Hayes SC, Lillis J. Acceptance and commitment therapy. Washington (DC): APA; 2012.

Heinonen E, Nissen-Lie HA. The professional and personal characteristics of effective psychotherapists: a systematic review. Psychother Res. 2020 Apr;30(4):417-32.

Hermer M, Röhrle B. Handbuch der therapeutischen Beziehung. Tübingen: dgvt-Verlag; 2008. Vol. 1: Allgemeiner Teil.

Horvath AO, Greenberg LS, editors. The working alliance: Theory, research, and practice. New York: John Wiley; 1994.

Horvath AO, Greenberg LS. Development and validation of the Working Alliance Inventory. J Couns Psychol. 1989;36(2):223-33.

Horvath AO. Research on the alliance: knowledge in search of a theory. Psychother Res. $2018 \mathrm{Jul}$; 28(4):499-516

Jacob G, Seebauer L. Schematherapie. Fallvideos zu Persönlichkeitsstörungen und Suizidalität. Weinheim: Beltz Video-Learning; 2013.

Johns RG, Barkham M, Kellett S, Saxon D. A systematic review of therapist effects: a critical narrative update and refinement to review. Clin Psychol Rev. 2019 Feb;67:78-93.

Kanfer FH, Reinecker H, Schmelzer D. Selbstmanagement-Therapie. 5th ed. Berlin: Springer; 2012. https://doi.org/10.1007/9783-642-19366-8.

Kazantzis N, Dattilio FM, McGinn LK, Newman CF, Persons JB, Radomsky AS. Defining the role and function of the therapeutic relationship in cognitive behavioral therapy: a modified Delphi panel. Int J Cogn Ther. 2018; 11(2):158-83.

Kiesler DJ. The 1982 interpersonal circle: A taxonomy for complementarity in human transactions. Psychol Rev. 1983;90(3):185-214.

Kivlighan DM, Marmarosh CL, Hilsenroth MJ Client and therapist therapeutic alliance, session evaluation, and client reliable change: a moderated actor-partner interdependence model. J Couns Psychol. 2014 Jan;61(1):1523.

Knox J. The harmful effects of psychotherapy: when the therapeutic alliance fails. Br J Psychother. 2019;35(2):245-62.

Köhler S, Guhn A, Betzler F, Stiglmayr C, Brakemeier EL, Sterzer P. Therapeutic self-disclosure within DBT, schema therapy, and CBASP: opportunities and challenges. Front Psychol. 2017 Nov;8:2073.
Kramer U, Kolly S, Berthoud L, Keller S, Preisig M, Caspar F, et al. Effects of motive-oriented therapeutic relationship in a ten-session general psychiatric treatment of borderline personality disorder: a randomized controlled trial. Psychother Psychosom. 2014;83(3): 176-86.

Kube T, Glombiewski JA, Rief W. Erwartungsfokussierte psychotherapeutische Interventionen bei Personen mit depressiver Symptomatik. Verhaltenstherapie. 2019;29(4):111.

Lairaiter AR. Konzeptuelle Aspekte und Gestal tungsmöglichkeiten der therapeutischen Beziehung. In: Hermer M, Röhrle B, editors. Handbuch der therapeutischen Beziehung. Tübingen: dgvt-Verlag; 2008. Vol. 2, p. 11491178.

Lammers $\mathrm{CH}$. Therapeutische Beziehung und Gesprächsführung. Weinheim: Beltz; 2017.

Laws HB, Constantino MJ, Sayer AG, Klein DN, Kocsis JH, Manber R, et al. Convergence in patient-therapist therapeutic alliance ratings and its relation to outcome in chronic depression treatment. Psychother Res. 2017 Jul; 27(4):410-24.

Linehan M. Cognitive-behavioral treatment of borderline personality disorder. New York: Guilford Press; 1993.

Luborsky L. Helping alliances in psychotherapy. In: Cleghhorn JL, editor. Successful psychotherapy. New York: Mazel; 1976. p. 92-116.

Lutz W, De Jong K, Rubel J. Patient-focused and feedback research in psychotherapy: where are we and where do we want to go? Psychother Res. 2015;25(6):625-32.

McCullough JP. Treating chronic depression with disciplined personal involvement: cognitive behavioral analysis system of psychotherapy (CBASP). Berlin: Springer; 2006.

McCullough JP. Treatment for chronic depression: Cognitive Behavioral Analysis System of Psychotherapy. New York: Guilford Press; 2000.

McCullough JP Jr. Treatment for chronic depression: cognitive behavioral analysis system of psychotherapy (CBASP). J Clin Psychol. 2003 Aug;59(8):833-46.

Norcross JC, Lambert MJ. Psychotherapy relationships that work III. Psychotherapy (Chic). 2018 Dec;55(4):303-15.

Norcross JC, Lambert MJ. Evidence-based therapist conributions. Psychotherapy relationships that work. Volume 1. 3rd ed. New York: Oxford University Press; 2019.

Orlinsky DE, Howard KI. A generic model of psychotherapy. Int J Eclect Psychother. 1987;6: 6-27.

Probst GH, Berger T, Flückiger C. Die Allianz als Prädiktor für den Therapieerfolg internetbasierter Interventionen bei psychischen Störungen: eine korrelative Metaanalyse. Verhaltenstherapie. 2019;29:182-95.

Reese RJ, Usher EL, Bowman DC, Norsworthy LA, Halstead JL, Rowlands SR, et al. Using client feedback in psychotherapy training: an analysis of its influence on supervision and counselor self-efficacy. Train Educ Prof Psychol. 2009;3:157-68.
Renner F, Jarrett RB, Vittengl JR, Barrett MS, Clark LA, Thase ME. Interpersonal problems as predictors of therapeutic alliance and symptom improvement in cognitive therapy for depression. J Affect Disord. 2012 May; 138(3):458-67.

Rief W, Glombiewski JA. Erwartungsfokussierte psychotherapeutische Interventionen (EFPI). Verhaltenstherapie. 2016;26:47-54.

Rief W, Strauß B. Integratives Störungsmodell als Grundlage der Therapieplanung. Psychotherapeut. 2018;63:401-8.

Rogers CR. A theory of therapy, personality, and interpersonal relationships, as developed in the client-centered framework. In: Koch S, editor. Psychology: a study of a science. New York: McGraw-Hill; 1959. Study 1, Vol. 3 : Formulations of the person and the social context. p. 184-256.

Rubel JA, Fisher AJ, Husen K, Lutz W. Translating Person-Specific Network Models into Personalized Treatments: Development and Demonstration of the Dynamic Assessment Treatment Algorithm for Individual Networks (DATA-IN). Psychother Psychosom. 2018;87(4):249-51.

Rubel J, Lutz W. Personalisierung statt Verfahrensinflation - Oder: Warum die Psychotherapieforschung in Deutschland ausgebaut werden sollte/könnte/müsste. Teil 2. Psychother Psychosom Med Psychol. 2016;66:463-4.

Sachse R. Persönlichkeitsstörungen: Psychotherapie dysfunktionaler Interaktionsstile. Göttingen: Hogrefe; 1997.

Sachse R. Therapeutische Beziehungsgestaltung. Göttingen: Hogrefe; 2006.

Sachse R, Fasbender J, Sachse M. Klärungsorientierte Psychotherapie von Persönlichkeitsstörungen: Grundlagen und Konzepte. Göttingen: Hogrefe; 2011.

Safran JD, Muran JC. Resolving therapeutic alliance ruptures: diversity and integration. J Clin Psychol. 2000 Feb;56(2):233-43.

Safran JD, Muran JC, Eubanks-Carter C. Repairing alliance ruptures. Psychotherapy (Chic). 2011 Mar;48(1):80-7.

Santiago NJ, Klein DN, Vivian D, Arnow BA, Blalock JA, Kocsis JH, et al. The therapeutic alliance and CBASP-specific skill acquisition in the treatment of chronic depression. Cognit Ther Res. 2005;29:803-17.

Schamong I, Bollmann S, Struck N, Kube T, D'Astolfo L, Brakemeier E-L. Insights into the formation of a trusting therapeutic relationship: feasibility of an experimental approach. Psychother Psychosom. 2019;88 (suppl):1145 .

Scheel MJ, Davis CK, Henderson JD. Therapist use of client strengths. Couns Psychol. 2013; 41:392-427.

Schiefele AK, Lutz W, Barkham M, Rubel J, Böhnke J, Delgadillo J, et al. Reliability of Therapist Effects in Practice-Based Psychotherapy Research: A Guide for the Planning of Future Studies. Adm Policy Ment Health. 2017 Sep; 44(5):598-613.

Schiepek GK, Aichhorn W, Schöller H. Monitoring change dynamics - a nonlinear approach to psychotherapy feedback. Chaos Complex Lett. 2018;11:355-75. 
Schneibel R, Scholz C, Brakemeier EL. Interpersonelle Psychotherapie. In: Brakemeier EL Jacobi F, editors. Verhaltenstherapie in der Praxis. Weinheim: Beltz; 2017. pp. 726-39.

Scholz W. Die therapeutische Beziehung. In: Sulz SK, editor. Therapie-Buch. Kognitiv-behaviorale Psychotherapie in Psychiatrie, Psychotherapeutischer Medizin und Klinischer Psychologie. München: CIP Medien; 1998. pp. 84-100.

Schöttke H, Flückiger C, Goldberg SB, Eversmann J, Lange J. Predicting psychotherapy outcome based on therapist interpersonal skills: A five-year longitudinal study of a therapist assessment protocol. Psychother Res. 2017 Nov;27(6):642-52.

Schramm E. Interpersonelle Psychotherapie. 4th ed. Stuttgart: Schattauer; 2019.

Schulte D. Therapieplanung. Göttingen: Hogrefe; 1996.

Schulte D, Eifert GH. What to do when manuals fail? The dual model of psychotherapy. Clin Psychol Sci Pract. 2002;9:312-28.

Sijercic I, Button ML, Westra HA, Hara KM. The interpersonal context of client motivational language in cognitive-behavioral therapy. Psychotherapy (Chic). 2016 Mar;53(1):1321.

Spinhoven P, Giesen-Bloo J, van Dyck R, Kooiman K, Arntz A. The therapeutic alliance in schema-focused therapy and transference-focused psychotherapy for borderline personality disorder. J Consult Clin Psychol. 2007 Feb; 75(1):104-15.
Stiglmayr C, Stecher-Mohr J, Wagner T, Meißner I, Spretz D, Steffens C, et al. Effectiveness of dialectic behavioral therapy in routine outpatient care: the Berlin Borderline Study. Borderline Personal Disord Emot Dysregul. 2014 Dec $; 1: 20$.

Wampold BE, Imel ZE. The great psychotherapy debate: the evidence for what makes psychotherapy work, Second Edi. New York: Routledge; 2015.

Wampold BE, Imel ZE, Flückiger C. Die Psychotherapie-Debatte [Internet]Göttingen: Hogrefe; 2018.

Weissman MM, Markowitz JC, Klerman GL. Comprehensive guide to interpersonal psychotherapy. New York: Basic Books; 2000.

Wolpe J. Reciprocal inhibition as the main basis of psychotherapeutic effects. AMA Arch Neurol Psychiatry. 1954 Aug;72(2):205-26.

World Health Organization. Child maltreatment fact sheet. Geneva: World Health Organization; 2016.

Young JE. Cognitive therapy for personality disorders: a schema-focused approach. Sarasota: Professional Resource Press; 1994.

Young JE, Klosko JS, Weishaar ME. Schema therapy: a practitioner's guide. New York: Guilford Press; 2003.

Zilcha-Mano S. Is the alliance really therapeutic? Revisiting this question in light of recent methodological advances. Am Psychol. 2017 May-Jun;72(4):311-25.
Zilcha-Mano S, Errázuriz P. One size does not fit all: examining heterogeneity and identifying moderators of the alliance-outcome association. J Couns Psychol. 2015 Oct;62(4):57991.

Zilcha-Mano S, Lipsitz I, Errázuriz P. When is it effective to focus on the alliance? Analysis of a within-client moderator. Cognit Ther Res. 2018a;42(2):159-71.

Zilcha-Mano S, Muran JC, Eubanks CF, Safran JD, Winston A. When therapist estimations of the process of treatment can predict patients rating on outcome: the case of the working alliance. J Consult Clin Psychol. 2018b Apr;86(4):398-402.

Zilcha-Mano S, Muran JC, Eubanks CF, Safran JD, Winston A. Not just a non-specific factor: moderators of the effect of within- and between-clients alliance on outcome in CBT. Cognit Ther Res. 2018c;42(2):146-58.

Zimmer D. Die therapeutische Beziehung. Konzepte, empirische Befunde und Prinzipien ihrer Gestaltung. Weinheim: Edition Psychologie; 1983a.

Zimmer D. Überlegungen zu einem Modell der Therapeut-Klient-Beziehung. In Zimmer D, editor. Therapeutische Beziehung. Konzepte, empirische Befunde und Prinzipien ihrer Gestaltung. Weinheim: Edition Psychologie; 1983b. p. 138-49.

Zimmer D, Zimmer FT. Das Konzept der funktionalen Beziehungsgestaltung in der Verhaltenstherapie. In: Reinecker HS, Schmelzer D, editors. Verhaltenstherapie, Selbstregulation, Selbstmanagement. Göttingen: Hogrefe; 1996. p. 131-43. 